\title{
Complex Evolutionary Systems in Behavioral Finance
}

\author{
contributed chapter to the Handbook of \\ Financial Markets: Dynamics and Evolution, \\ T. Hens and K.R. Schenk-Hoppé (Eds.) \\ Cars Hommes and Florian Wagener \\ CeNDEF, School of Economics, University of Amsterdam
}





\section{CONTENTS}

1 Complex Evolutionary Systems in Behavioral Finance 5

1.1 An asset pricing model with heterogeneous beliefs . . . . . . . . . 8

1.1.1 The fundamental benchmark with rational agents . . . . . . . 9

1.1.2 Heterogeneous beliefs . . . . . . . . . . . . . . 10

1.1.3 Evolutionary dynamics . . . . . . . . . . . . . . 11

1.1.4 Forecasting rules ... . . . . . . . . . . . . . 13

1.2 Simple examples . . . . . . . . . . . . . . . . . . 14

1.2.1 Costly fundamentalists versus trend followers . . . . . . . . . 15

1.2.2 Fundamentalists versus opposite biases . . . . . . . . . . . . 17

1.2.3 Fundamentalists versus trend and bias . . . . . . . . . . . 18

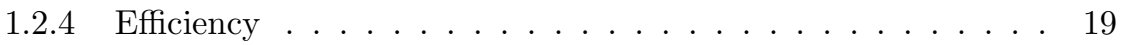

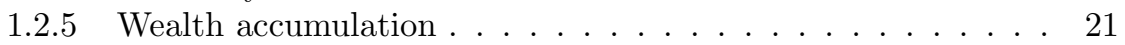

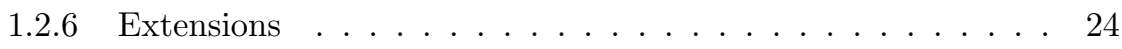

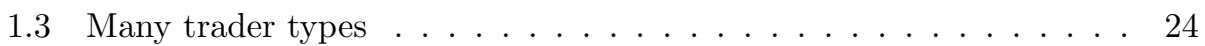

1.4 Empirical validation . . . . . . . . . . . . . . . . . . 29

1.4.1 The model in price-to-cash flows . . . . . . . . . . . . . 30

1.4.2 Estimation of a simple two-type example . . . . . . . . . . 34

1.4.3 Empirical implications . . . . . . . . . . . . . . . . 38

1.5 Laboratory experiments . . . . . . . . . . . . . . . . . . . . . . . . . . . 41

1.5.1 Learning to forecast experiments . . . . . . . . . . . . . . 42

1.5.2 The price generating mechanism . . . . . . . . . . . . 44

1.5.3 Benchmark expectations rules . . . . . . . . . . . . 45

1.5.4 Aggregate behavior . . . . . . . . . . . . . . . . 46

1.5.5 Individual prediction strategies . . . . . . . . . . . . . . 48

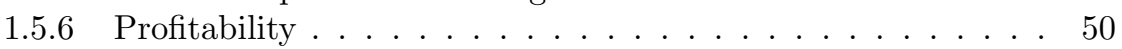

1.6 Concluding remarks . . . . . . . . . . . . . . . . . . . 52

A.1 Basic concepts from dynamical systems. . . . . . . . . . . . . . 54

A.2 Bifurcation scenarios . . . . . . . . . . . . . 56

$\begin{array}{ll}\text { Bibliography } & 59\end{array}$ 



\title{
COMPLEX EVOLUTIONARY SYSTEMS IN BEHAVIORAL FINANCE
}

\begin{abstract}
Traditional finance is built on the rationality paradigm. This chapter discusses simple models from an alternative approach in which financial markets are viewed as complex evolutionary systems. Agents are boundedly rational and base their investment decisions upon market forecasting heuristics. Prices and beliefs about future prices co-evolve over time with mutual feedback. Strategy choice is driven by evolutionary selection, so that agents tend to adopt strategies that were successful in the past. Calibration of "simple complexity models" with heterogeneous expectations to real financial market data and laboratory experiments with human subjects are also discussed.
\end{abstract}

Finance is witnessing important changes, according to some even a paradigmatic shift, from the traditional, neoclassical mathematical modeling approach based on a representative, fully rational agent and perfectly efficient markets (Muth (1961), Lucas (1971), Fama (1970)) to a behavioral approach based on computational models where markets are viewed as complex evolving systems with many interacting, "boundedly rational" agents using simple "rule of thumb" trading strategies (e.g. Anderson et al. (1988), Brock (1993), Arthur (1995), Arthur et al. (1997a), Tesfatsion and Judd (2006)). Investor's psychology plays a key role in behavioral finance, and different types of psychology based trading and behavioral modes have been identified in the literature, such as positive feedback or momentum trading, trend extrapolation, noise trading, overconfidence, overreaction, optimistic or pessimistic traders, upward or downward biased traders, correlated imperfect rational trades, overshooting, contrarian strategies, etc.. Some key references dealing with various aspects of investor psychology include e.g. Cutler et al. (1990), DeBondt and Thaler (1985), DeLong et al. (1990a, 1990b), Brock and Hommes (1997, 1998), 
Gervais and Odean (2001) and Hong and Stein (1999, 2003), among others; see e.g. Shleifer (2000), Hirshleifer (2001) and Barberis and Thaler (2003) for extensive surveys and many more references on behavioral finance.

An important problem of a behavioral approach is that it leaves "many degrees of freedom". There are many ways individual agents can deviate from full rationality. Evolutionary selection based on relative performance is one plausible way to discipline the "wilderness of bounded rationality". Milton Friedman (1953) has argued that non-rational agents will not survive evolutionary competition and will therefore be driven out of the market, thus providing support to a representative rational agent framework as a (long run) description of the economy. In the same spirit, Alchian (1950) argued that biological evolution and natural selection driven by realized profits may eliminate non-rational, non-optimizing firms and lead to a market where rational, profit maximizing firms dominate. Blume and Easley $(1992,2006)$ have shown however that the market selection hypothesis does not always hold and that non-rational agents may survive in the market. Brock (1993,1997), Arthur et al. (1997b), LeBaron et al. (1999) and Farmer (2002), amongst others, introduced artificial stock markets, described by agent based models with evolutionary selection among many different interacting trading strategies. They showed that the market does not generally select for the rational, fundamental strategy, and that simple technical trading strategies may survive in artificial markets. Computationally oriented agent-based simulation models have been reviewed in LeBaron (2006); see also the special issue of the Journal of Mathematical Economics (Hens and Schenk-Hoppé, 2005) and the survey chapter of Evstigneev, Hens and Schenk-Hoppé (2009) in this Handbook for an overview of evolutionary finance ${ }^{1}$.

Stimulated by work on artificial markets, in the last decade quite a number of "simple complexity models" have been introduced. Markets are viewed as evolutionary adaptive systems with boundedly rational interacting agents, but the models are simple enough to be at least partly analytically tractable. The study of simple complexity models typically requires a well balanced mixture of analytical and computational tools. This literature is surveyed in Hommes (2006) and Chiarella (2007); see also Lux (2009), who discusses in detail how well models with interacting agents match important stylized facts such as fat tails in the returns distribution and long memory. Without repeating an extensive survey, this chapter focuses on a number of simple examples, in particular the adaptive belief systems (ABS) of Brock and Hommes $(1997,1998)$. These models serve as didactic examples of nonlinear dynamic asset pricing models with evolutionary strategy switching and they illustrate some of the key features present in the interacting agents literature. The model also has been used to test the relevance of the theory of heterogeneous expectations empirically as well as in laboratory experiments with human subjects. Simple complexity models may also be used by practitioners or policy makers. To

\footnotetext{
${ }^{1}$ Some other recent references are Amir et al. (2005) and Evstigneev et al. (2002, 2008).
} 
illustrate this point, we present an example how such a model can be used to evaluate how likely it is that a stock market bubble will resume.

Two important features of the ABS are that agents are boundedly rational and that they have heterogeneous expectations. An ABS is in fact a standard discounted value asset pricing model derived from mean-variance maximization, extended to the case of heterogeneous beliefs. Two classes of investors that are also be observed in financial practice, can be distinguished: fundamentalists and technical analysts. Fundamentalists base their forecasts of future prices and returns upon economic fundamentals, such as dividends, interest rates, price-earning ratio's, etc. In contrast, technical analysts are looking for patterns in past prices and base their forecasts upon extrapolation of these patterns. Fractions of these two types of traders are time varying and depend upon relative performance. Strategy choice is thus based on evolutionary selection or reinforcement learning, with agents switching to more successful (i.e. profitable) rules. Asset price fluctuations are characterized by irregular switching between a stable phase when fundamentalists dominate the market and an unstable phase when trend followers dominate and asset prices deviate from benchmark fundamentals. Price deviations from the rational expectations fundamental and excess volatility are triggered by news about economic fundamentals but may be amplified by evolutionary selection of trend following strategies.

There is empirical evidence that experience based reinforcement learning plays an important role in investment decisions in real markets. For example, Ippolito (1992), Chevalier and Ellison (1997), Sirri and Tufano (1998), Rockinger (1996) and Karceski (2002) show for mutual funds data that money flows into past good performers, while flowing out of past poor performers, and that performance persists on a short term basis. Pension funds are less extreme in picking good performance but are tougher on bad performers (Del Guercio and Tkac, 2002). Benartzi and Thaler (2007) have shown that heuristics and biases play a significant role in retirement savings decisions. For example, using data from Vanguard they show that the equity allocation of new participants rose from $58 \%$ in 1992 to $74 \%$ in 2000 , following a strong rise in stock prices in the late 1990s, but dropped back to $54 \%$ in 2002, following the strong fall in stock prices.

Laboratory experiments with human subjects have shown that individuals often do not behave fully rational, but tend to use heuristics, possibly biased, in making economic decisions under uncertainty (Kahneman and Tversky, 1974). In a similar vein, Smith et al. (1988) have shown the occurrence of bubbles and the ease with which markets deviate from full rationality in asset pricing laboratory experiments. These bubbles occur despite the fact that participants had sufficient information to compute the fundamental value of the asset. Laboratory experiments with human subjects provide an important tool to investigate which behavioral rules lay a significant role in deviations from the rational benchmark, and they can thus help to discipline the class of behavioral modes. Duffy (2007) gives a stimulating recent overview concerning the role of laboratory experiments to explain macro phenomena. 
Heterogeneity in forecasting future asset prices is supported by evidence from survey data. For example, Vissing-Jorgensen (2003) reports that at the beginning of $2000,50 \%$ of individual investors considered the stock market to be overvalued, approximately $25 \%$ believed that it was fairly valued, about $15 \%$ were unsure and less than $10 \%$ believed that it was undervalued. This is an indication of heterogeneous beliefs among individual investors about the prospect of the stock market. Similarly, Shiller (2000) finds evidence that investors' sentiment varies over time. Both institutional and individual investors become more optimistic in response to significant increases in the recent performance of the stock market.

This chapter is organized as follows. Section 1.1 introduces the main features of adaptive belief systems and Section 1.2 discusses a number of simple examples with 2, 3 and 4 different trader types. In Section 1.3 an analytical framework with many different trader types is presented. Section 1.4 discusses the empirical relevance of behavioral heterogeneity. The estimation of a simple model with fundamentalists and chartist on yearly S\&P500 data shows how the worldwide stock market bubble in the late 1990s, triggered by good news about fundamentals (a new, internet technology), may have been strongly amplified by trend following strategies. Section 1.5 reviews some learning to forecast laboratory experiments with human subjects, investigating which individual forecasting rules agents may use, how these rules interact and which aggregate outcome they co-create. Section 1.6 concludes, sketching some challenges for future research and potential applications for financial practitioners and policy makers. An appendix contains a short mathematical overview of bifurcation theory, which plays a role in the transition to complicated price fluctuations in the simple complexity models discussed in this chapter.

\subsection{AN ASSET PRICING MODEL WITH HETEROGENEOUS BELIEFS}

This section discusses the asset pricing model with heterogeneous beliefs as introduced in Brock and Hommes (1998), using evolutionary selection of expectations as in Brock and Hommes (1997a). This simple modeling framework has been inspired by computational work at the Santa Fe Institute (SFI) and may be viewed as a simple, partly analytically tractable, version of the more complicated SFI artificial stock market of Arthur et al. (1997b).

Agents can either invest in a risk free or in a risky asset. The risk free asset is in perfect elastic supply and pays a fixed rate of return $r$; the risky asset pays an uncertain dividend. Let $p_{t}$ be the price per share (ex-dividend) of the risky asset at time $t$, and let $y_{t}$ be the stochastic dividend process of the risky asset. Wealth dynamics is given by

$$
W_{t+1}=R W_{t}+\left(p_{t+1}+y_{t+1}-R p_{t}\right) z_{t},
$$

where $R=1+r$ is the gross rate of risk free return and $z_{t}$ denotes the number of shares of the risky asset purchased at date $t$. Let $E_{h t}$ and $V_{h t}$ denote the 'beliefs' or 
forecasts of trader type $h$ about conditional expectation and conditional variance. Agents are assumed to be myopic mean-variance maximizers so that the demand $z_{h t}$ of type $h$ for the risky asset solves

$$
\operatorname{Max}_{z_{t}}\left\{E_{h t}\left[W_{t+1}\right]-\frac{a}{2} V_{h t}\left[W_{t+1}\right]\right\}
$$

where $a$ is the risk aversion parameter. The demand $z_{h t}$ for risky assets by trader type $h$ is then

$$
z_{h t}=\frac{E_{h t}\left[p_{t+1}+y_{t+1}-R p_{t}\right]}{a V_{h t}\left[p_{t+1}+y_{t+1}-R p_{t}\right]}=\frac{E_{h t}\left[p_{t+1}+y_{t+1}-R p_{t}\right]}{a \sigma^{2}},
$$

where the conditional variance $V_{h t}=\sigma^{2}$ is assumed to be constant and equal for all types. ${ }^{2}$ Let $z^{s}$ denote the supply of outside risky shares per investor, also assumed to be constant, and let $n_{h t}$ denote the fraction of type $h$ at date $t$. Equilibrium of demand and supply yields

$$
\sum_{h=1}^{H} n_{h t} \frac{E_{h t}\left[p_{t+1}+y_{t+1}-R p_{t}\right]}{a \sigma^{2}}=z^{s},
$$

where $H$ is the number of different trader types. The forecasts $E_{h t}\left[p_{t+1}+y_{t+1}\right]$ of tomorrows prices and dividends are made before the equilibrium price $p_{t}$ has been revealed by the market and therefore will depend upon a publically available information set $I_{t-1}=\left\{p_{t-1}, p_{t-2}, \ldots ; y_{t-1}, y_{t-2}, \ldots\right\}$ of past prices and dividends. Solving the heterogeneous market clearing equation for the equilibrium price gives

$$
R p_{t}=\sum_{h=1}^{H} n_{h t} E_{h t}\left[p_{t+1}+y_{t+1}\right]-a \sigma^{2} z^{s} .
$$

The quantity $a \sigma^{2} z^{s}$ may be interpreted as a risk premium for traders to hold risky assets.

\subsubsection{The fundamental benchmark with rational agents}

When all agents are identical and expectations are homogeneous the equilibrium pricing equation (1.1.5) reduces to

$$
R p_{t}=E_{t}\left[p_{t+1}+y_{t+1}\right]-a \sigma^{2} z^{s}
$$

where $E_{t}$ is the common conditional expectation in the beginning of period $t$. It is well known that, assuming that a transversality condition $\lim _{t \rightarrow \infty}\left(E_{t}\left[p_{t+k}\right]\right) / R^{k}=0$

\footnotetext{
${ }^{2}$ Gaunersdorfer (2000) investigates the case with time varying beliefs about variances and shows that the asset price dynamics are quite similar. Chiarella and $\mathrm{He}(2002,2003)$ investigate the model with heterogeneous risk aversion coefficients.
} 
holds, the price of the risky asset is given by the discounted sum of expected future dividends minus the risk premium:

$$
p_{t}^{*}=\sum_{k=1}^{\infty} \frac{E_{t}\left[y_{t+k}\right]-a \sigma^{2} z^{s}}{R^{k}} .
$$

The price $p_{t}^{*}$ in (1.1.7) is called the fundamental rational expectations price, or the fundamental price for short. It is completely determined by economic fundamentals, which are here given by the stochastic dividend process $y_{t}$. In this Section we will focus on the case of an independently identically distributed (IID) dividend process $y_{t}$, but the estimation of the simple 2-type model discussed in Section 1.4 uses a non-stationary dividend process ${ }^{3}$. For the special case of an IID dividend process $y_{t}$, with constant mean $E\left[y_{t}\right]=\bar{y}$, the fundamental price is constant:

$$
p^{*}=\sum_{k=1}^{\infty} \frac{\bar{y}-a \sigma^{2} z^{s}}{R^{k}}=\frac{\bar{y}-a \sigma^{2} z^{s}}{r} .
$$

Recall that, in addition to the rational expectations fundamental solution (1.1.7), so-called rational bubble solutions of the form $p_{t}=p_{t}^{*}+(1+r)^{t}\left(p_{0}-p_{0}^{*}\right)$ also satisfy the pricing equation (1.1.6). Along these bubble solutions, traders have rational expectations (perfect foresight), but they are ruled out by the transversality condition. In a perfectly rational world, traders realize that such bubbles cannot last forever and therefore all traders believe that the value of a risky asset equals its fundamental price forever. Changes in asset prices are then only driven by unexpected changes in dividends and random 'news' about economic fundamentals. In a heterogeneous world the situation will however be quite different.

\subsubsection{Heterogeneous beliefs}

It will be convenient to work with the deviation from the fundamental price

$$
x_{t}=p_{t}-p_{t}^{*}
$$

We make the following assumptions about the beliefs of trader type $h$ :

B1 $V_{h t}\left[p_{t+1}+y_{t+1}-R p_{t}\right]=V_{t}\left[p_{t+1}+y_{t+1}-R p_{t}\right]=\sigma^{2}$, for all $h, t$.

B2 $E_{h t}\left[y_{t+1}\right]=E_{t}\left[y_{t+1}\right]=\bar{y}$, for all $h, t$.

B3 All beliefs $E_{h t}\left[p_{t+1}\right]$ are of the form

$$
E_{h t}\left[p_{t+1}\right]=E_{t}\left[p_{t+1}^{*}\right]+E_{h t}\left[x_{t+1}\right]=p^{*}+f_{h}\left(x_{t-1}, \ldots, x_{t-L}\right), \quad \text { for all } h, t .
$$

\footnotetext{
${ }^{3}$ Brock and Hommes (1997b) also discuss a non-stationary example, where the dividend process follows a geometric random walk.
} 
According to B1 beliefs about conditional variance are equal and constant for all types, as discussed above already. Assumption B2 states that all types have correct expectations about future dividends $y_{t+1}$ given by the conditional expectation, which is $\bar{y}$ in the case of IID dividends. According to B3, beliefs about future prices consist of two parts: a common belief about the fundamental plus a heterogeneous part $f_{h t}{ }^{4}$. Each forecasting rule $f_{h}$ represents a model of the market (e.g. a technical trading rule) according to which type $h$ believes that prices will deviate from the fundamental price.

An important and convenient consequence of the assumptions B1-B3 about traders' beliefs is that the heterogeneous agent market equilibrium equation (1.1.5) can be reformulated in deviations from the benchmark fundamental. In particular substituting the price forecast (1.1.10) in the market equilibrium equation (1.1.5) and using $R p_{t}^{*}=E_{t}\left[p_{t+1}^{*}+y_{t+1}\right]-a \sigma^{2} z^{s}$ yields the equilibrium equation in deviations from the fundamental:

$$
R x_{t}=\sum_{h=1}^{H} n_{h t} E_{h t}\left[x_{t+1}\right] \equiv \sum_{h=1}^{H} n_{h t} f_{h t}
$$

with $f_{h t}=f_{h}\left(x_{t-1}, \ldots, x_{t-L}\right)$. Note that the benchmark fundamental is nested as a special case within this general setup, with all forecasting strategies $f_{h} \equiv 0$. Hence, the adaptive belief systems can be used in empirical and experimental testing whether asset prices deviate significantly from some benchmark fundamental.

\subsubsection{Evolutionary dynamics}

The evolutionary part of the model describes how beliefs are updated over time, that is, how the fractions $n_{h t}$ of trader types evolve over time. These fractions are updated according to an evolutionary fitness or performance measure. The fitness measures of all trading strategies are publically available, but subject to noise. Fitness is derived from a random utility model and given by

$$
\tilde{U}_{h t}=U_{h t}+\varepsilon_{i h t},
$$

where $U_{h t}$ is the deterministic part of the fitness measure and $\varepsilon_{i h t}$ represents an individual agent's IID error when perceiving the fitness of strategy $h=1, \ldots H$. In order to obtain analytical expressions for the probabilities or fractions, the noise term $\varepsilon_{i h t}$ is assumed to be drawn from a double exponential distribution. As the number of agents goes to infinity, the probability that an agent chooses strategy $h$

\footnotetext{
${ }^{4}$ The assumption that all types know the fundamental price is without loss of generality, because any forecasting rule not using the fundamental price can be re-parameterized or reformulated for mathematical convenience in deviations from an (unknown) fundamental price $p^{*}$.
} 
is then given by the multi-nomial logit model (or 'Gibbs' probabilities) ${ }^{5}$

$$
n_{h t}=\frac{e^{\beta U_{h, t-1}}}{\sum_{h=1}^{H} e^{\beta U_{h, t-1}}} .
$$

Note that the fractions $n_{h t}$ add up to 1. A key feature of (1.1.13) is that the higher the fitness of trading strategy $h$, the more traders will select strategy $h$. Hence, (1.1.13) represents a form of reinforcement learning: agents tend to switch to strategies that have performed well in the (recent) past. The parameter $\beta$ in (1.1.13) is called the intensity of choice; it measures the sensitivity of the mass of traders to selecting the optimal prediction strategy. The intensity of choice $\beta$ is inversely related to the variance of the noise terms $\varepsilon_{i h t}$. The extreme case $\beta=0$ corresponds to noise of infinite variance, so that differences in fitness cannot be observed and all fractions (1.1.13) will be fixed over time and equal to $1 / H$. The other extreme case $\beta=+\infty$ corresponds to the case without noise, so that the deterministic part of the fitness can be observed perfectly and in each period, all traders choose the optimal forecast. An increase in the intensity of choice $\beta$ represents an increase in the degree of rationality with respect to evolutionary selection of trading strategies. The timing of the coupling between the market equilibrium equation (1.1.5) or (1.1.11) and the evolutionary selection of strategies (1.1.13) is important. The market equilibrium price $p_{t}$ in (1.1.5) depends upon the fractions $n_{h t}$. The notation in (1.1.13) stresses the fact that these fractions $n_{h t}$ depend upon most recently observed past fitnesses $U_{h, t-1}$, which in turn depend upon past prices $p_{t-1}$ and dividends $y_{t-1}$ in periods $t-1$ and further in the past, as will be seen below. After the equilibrium price $p_{t}$ has been revealed by the market, it will be used in evolutionary updating of beliefs and determining the new fractions $n_{h, t+1}$. These new fractions will then determine a new equilibrium price $p_{t+1}$, etc. In an adaptive belief system, market equilibrium prices and fractions of different trading strategies thus co-evolve over time.

A natural candidate for evolutionary fitness is (a weighted average of) realized

\footnotetext{
${ }^{5}$ See Manski and McFadden (1981) and Anderson, de Palma and Thisse (1993) for extensive discussion of discrete choice models and their applications in economics.
} 
profits, given by ${ }^{6}$

$$
U_{h t}=\left(p_{t}+y_{t}-R p_{t-1}\right) \frac{E_{h, t-1}\left[p_{t}+y_{t}-R p_{t-1}\right]}{a \sigma^{2}}+w U_{h, t-1},
$$

where $0 \leq w \leq 1$ is a memory parameter measuring how fast past realized fitness is discounted for strategy selection.

Fitness can be rewritten in terms of deviations from the fundamental as

$$
U_{h t}=\left(x_{t}-R x_{t-1}+a \sigma^{2} z^{s}+\delta_{t}\right)\left(\frac{f_{h, t-1}-R x_{t-1}+a \sigma^{2} z^{s}}{a \sigma^{2}}\right)+w U_{h, t-1},
$$

where $\delta_{t} \equiv p_{t}^{*}+y_{t}-E_{t-1}\left[p_{t}^{*}+y_{t}\right]$ is a martingale difference sequence.

\subsubsection{Forecasting rules}

To complete the model we have to specify the class of forecasting rules. Brock and Hommes (1998) have investigated evolutionary competition between simple linear forecasting rules with only one lag, i.e.

$$
f_{h t}=g_{h} x_{t-1}+b_{h}
$$

It can be argued that, for a forecasting rule to have any impact in real markets, it has to be simple, because it seems unlikely that enough traders will coordinate on a complicated rule. The simple linear rule (1.1.16) includes a number of important special cases. For example, when both the trend and the bias parameters $g_{h}=$ $b_{h}=0$ the rule reduces to the fundamentalists forecast, i.e.

$$
f_{h t} \equiv 0,
$$

predicting that the deviation $x$ from the fundamental will be 0 , or equivalently that the price will be at its fundamental value. Other important cases covered by the linear forecasting rule (1.1.16) are the pure trend followers

$$
f_{h t}=g_{h} x_{t-1}, \quad g_{h}>0
$$

6 Note that this fitness measure does not take into account the risk taken at the moment of the investment decision. In fact, one could argue that the fitness measure (1.1.14) does not take into account the variance term in (1.1.2) capturing the investors' risk taken before obtaining that profit. On the other hand, in real markets realized net profits or accumulated wealth may be what investors care about most, and the non-risk adjusted fitness measure (1.1.14) may thus be of relevant in practice. See also DeLong et al. (1990) for a discussion of this point. Given that investors are risk averse mean-variance maximizers maximizing their expected utility from wealth (1.1.2), an alternative, natural candidate for fitness are the risk adjusted profits given by $\pi_{h t}=R_{t} z_{h, t-1}-\frac{a}{2} \sigma^{2} z_{h, t-1}^{2}$, where $R_{t}=p_{t}+y_{t}-R p_{t-1}$ and $z_{h, t-1}=E_{h, t-1}\left[R_{t}\right] /\left(a \sigma^{2}\right)$ is the demand by trader type $h$. Hommes (2001) shows that the risk adjusted fitness measure is, up to a type independent level, equivalent to minus squared prediction errors. 
and the pure biased belief

$$
f_{h t}=b_{h} .
$$

Notice that the simple pure bias forecast (1.1.19) represents any positively or negatively biased forecast of next periods price that traders might have. Instead of these extremely simple habitual rule of thumb forecasting rules, some might prefer the rational, perfect foresight forecasting rule

$$
f_{h t}=x_{t+1} \text {. }
$$

We emphasize however, that the perfect foresight forecasting rule (1.1.20) assumes perfect knowledge of the heterogeneous market equilibrium equation (1.1.5), and in particular perfect knowledge about the beliefs of all other traders. Although the case with perfect foresight has much theoretical appeal, its practical relevance in a complex heterogeneous world should not be overstated since this underlying assumption seems rather strong ${ }^{7}$.

\subsection{SIMPLE EXAMPLES}

This section presents simple but typical examples of adaptive belief systems (ABS), with two, three resp. four competing linear forecasting rules (1.1.16), where the parameter $g_{h}$ represents a perceived trend in prices and the parameter $b_{h}$ represents a perceived upward or downward bias. The ABS with $H$ types is given by (in deviations from the fundamental benchmark):

$$
\begin{aligned}
(1+r) x_{t} & =\sum_{h=1}^{H} n_{h t}\left(g_{h} x_{t-1}+b_{h}\right)+\epsilon_{t} \\
n_{h, t} & =\frac{e^{\left(\beta U_{h, t-1}\right)}}{\sum_{h=1}^{H} e^{\left(\beta U_{h, t-1}\right)}} \\
U_{h, t-1} & =\left(x_{t-1}-R x_{t-2}\right)\left(\frac{g_{h} x_{t-3}+b_{h}-R x_{t-2}}{a \sigma^{2}}\right)+w U_{h, t-2}-C_{h},
\end{aligned}
$$

where $\epsilon_{t}$ is a small noise term representing, for example, a small fraction of noise traders and/or random outside supply of the risky asset. In order to keep the analysis of the dynamical behavior tractable, Brock and Hommes (1998) focused on the case where the memory parameter $w=0$, so that evolutionary fitness is given by last period's realized profit. A common feature of all examples is that, as the intensity of choice to switch prediction or trading strategies increases, the

\footnotetext{
${ }^{7}$ Brock and Hommes (1997) analyze the cobweb model with costly rational versus cheap naive expectations, and find irregular price fluctuations due to endogenous switching between free riding and costly rational forecasting. In general however, a temporary equilibrium model with heterogeneous beliefs such as the asset pricing model, is difficult to analyze if one of the types has perfect foresight. In a recent paper, Brock et al. (2008) discuss how a perfect foresight trader may affect the dynamics in an asset pricing model with heterogeneous beliefs.
} 
fundamental steady state becomes locally unstable and non-fundamental steady states, cycles or even chaos arise. In the examples below, we will encounter different bifurcation routes (i.e. transitions) to complicated dynamics. A mathematical appendix summarizes the most important bifurcations, that is, qualitative changes in the dynamics (e.g. when a steady state loses stability or a new cycle is created) when a model parameter changes.

\subsubsection{Costly fundamentalists versus trend followers}

The simplest example of an ABS only has two trader types, with forecasting rules

$$
\begin{aligned}
& f_{1 t}=0 \quad \text { fundamentalists } \\
& f_{2 t}=g x_{t-1}, \quad g>0, \quad \text { trend followers. }
\end{aligned}
$$

The first type are fundamentalists predicting that the price will equal its fundamental value (or equivalently that the deviation will be zero) and the second type are pure trend followers predicting that prices will rise (or fall) by a constant rate. In this example, the fundamentalists have to pay a fixed per period positive $\operatorname{cost} C_{1}$ for information gathering; in all other examples discussed below information costs will be set to zero for all trader types.

For small values of the trend parameter, $0 \leq g<1+r$, the fundamental steady state is always stable. Only for sufficiently high trend parameters, $g>1+r$, trend followers can destabilize the system. For trend parameters, $1+r<g<(1+r)^{2}$ the dynamic behavior of the evolutionary system depends upon the intensity of choice to switch between the two trading strategies ${ }^{8}$. For low values of the intensity of choice, the fundamental steady state will be stable. As the intensity of choice increases, the fundamental steady state becomes unstable due to a pitchfork bifurcation in which two additional non-fundamental steady states $-x^{*}<0<x^{*}$ are created. As the intensity of choice increases further, the two non-fundamental steady states also become unstable due to a Hopf-bifurcation, and limit cycles or even strange attractors can arise around each of the (unstable) non-fundamental steady states ${ }^{9}$. The evolutionary ABS may cycle around the positive non-fundamental steady state, cycle around the negative non-fundamental steady state or, driven by the noise, switch back and forth between cycles around the high and the low steady state, as illustrated in Figure 1.1.

This example shows that, in the presence of information costs and with zero memory, when the intensity of choice in evolutionary switching is high fundamentalists

\footnotetext{
${ }^{8}$ For $g>(1+r)^{2}$ the system may become globally unstable and prices may diverge to infinity. Imposing a stabilizing force, for example by assuming that trend followers condition their rule upon deviations from the fundamental e.g. as in Gaunersdorfer, Hommes and Wagener (2008), leads to a bounded system again, possibly with cycles or even chaotic fluctuations.

${ }^{9}$ See the appendix for a more detailed discussion of the pitchfork bifurcation and the Hopf bifurcation.
} 

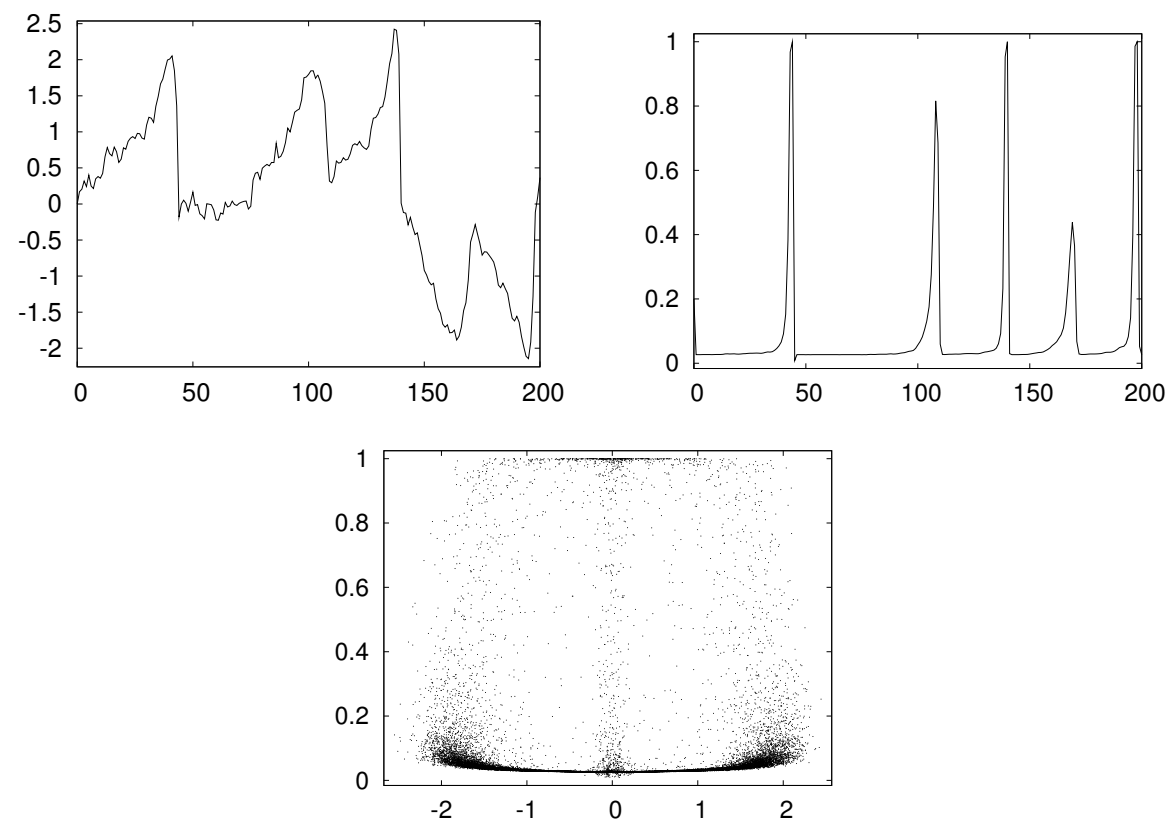

Figure 1.1. Time series of price deviations from fundamental (top left) and fractions of fundamentalists (top right) and attractor (bottom) in the $\left(x_{t}, n_{1 t}\right)$-phase space, for 2-type model with costly fundamentalist versus trend followers buffeted with small noise $(S D=0.1)$. The price dynamics is characterized by temporary bubbles when trend followers dominate the market, interrupted by sudden crashes when fundamentalists dominate. In the presence of (small) noise, the system switches back and forth between two co-existing quasi-periodic attractors of the underlying deterministic skeleton, one with prices above and one with prices below its fundamental value. Parameters are: $\beta=3.6, g=1.2, R=1.1$ and $C=1$.

can not drive out pure trend followers and persistent deviations from the fundamental price may occur. ${ }^{10}$

Figure 1.2 illustrates that the asset pricing model with costly fundamentalists versus cheap trend following exhibits a rational route to randomness, that is, a bifurcation route to chaos occurs as the intensity of choice to switch strategies increases.

\footnotetext{
${ }^{10}$ Brock and Hommes (1999) show that this result also holds when the memory in the fitness measure increases. In fact, an increase in the memory of the evolutionary fitness leads to bifurcation routes very similar to bifurcation routes due to an increase in the intensity of choice.
} 

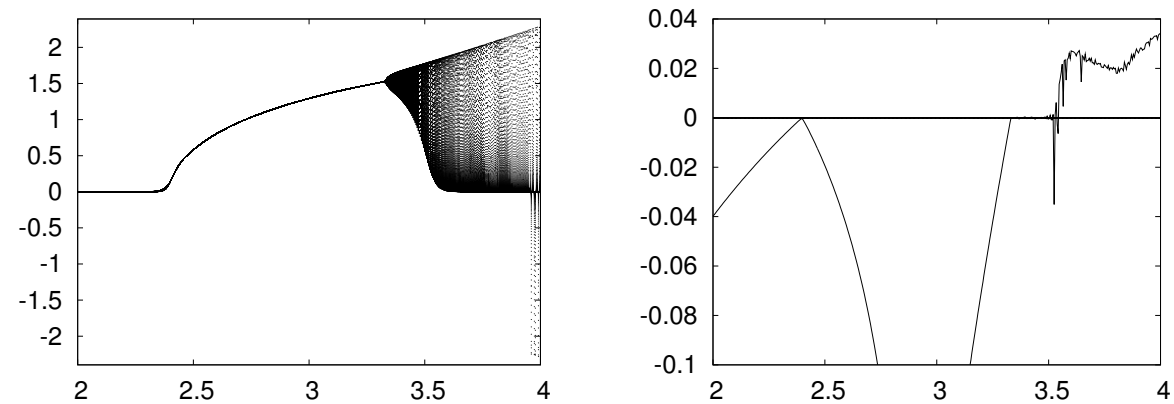

Figure 1.2. Bifurcation diagram (left) and largest Lyapunov exponent plot (right) as a function of the intensity of choice $\beta$ for 2-type model with costly fundamentalist versus trend followers. In both plots the model is buffeted with very small noise $\left(S D=10^{-6}\right.$ for the noise term $\epsilon_{t}$ in (1.2.1)), to avoid that for large $\beta$-values the system gets stuck in the locally unstable steady state. Parameters are: $g=1.2, R=1.1, C=1$ and $2 \leq \beta \leq 4$. A pitchfork bifurcation of the fundamental steady state, in which two stable non-fundamental steady states are created, occurs for $\beta \approx 2.37$. The non-fundamental steady states become unstable due to a Hopf-bifurcation for $\beta \approx 3.33$, and (quasi-)periodic dynamics arises. For large values of $\beta$ the largest Lyapunov exponent becomes positive indicating chaotic price dynamics.

\subsubsection{Fundamentalists versus opposite biases}

The second example of an ABS is an example with three trader types without any information costs. The forecasting rules are

$$
\begin{array}{lrlrl}
f_{1 t} & =0 & & \text { fundamentalists } \\
f_{2 t}=b & b>0, & & \text { positive bias (optimists) } \\
f_{3 t} & =-b & -b<0, & & \text { negative bias (pessimists). }
\end{array}
$$

The first type are fundamentalists as before, but there are no information costs for fundamentalists. The second and third types have a purely biased belief, expecting a constant price above respectively below the fundamental price.

For low values of the intensity of choice, the fundamental steady state is stable. As the intensity of choice increases the fundamental steady becomes unstable due to a Hopf bifurcation and the dynamics of the ABS is characterized by cycles around the unstable steady state. This example shows that, even when there are no information costs for fundamentalists, they cannot drive out other trader types with opposite biased beliefs. In the evolutionary ABS with high intensity of choice, fundamentalists and biased traders co-exist with fractions varying over time and prices fluctuating around the unstable fundamental steady state. Moreover, Brock and Hommes (1998, p.1259, lemma 9) show that as the intensity of choice tends 
to infinity the ABS converges to a (globally) stable cycle of period 4. Average profits along this 4-cycle are equal for all three trader types. Hence, if the initial wealth is equal for all three types, then in this evolutionary system in the long run accumulated wealth will be equal for all three types. This example shows that the Friedman argument that smart-fundamental traders will always drive out simple rule of thumb speculative traders is in general not valid ${ }^{11}$.

\subsubsection{Fundamentalists versus trend and bias}

The third example of an ABS is an example with four trader types, with linear forecasting rules (1.1.16) with parameters $g_{1}=0, b_{1}=0 ; g_{2}=0.9, b_{2}=0.2$; $g_{3}=0.9, b_{3}=-0.2$ and $g_{4}=1+r=1.01, b_{4}=0$. The first type are fundamentalists again, without information costs, and the other three types follow a simple linear forecasting rule with one lag. The dynamical behaviour is illustrated in Figures 1.3 and 1.4 .

(a)

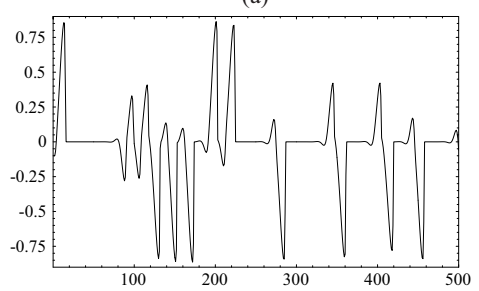

(c)

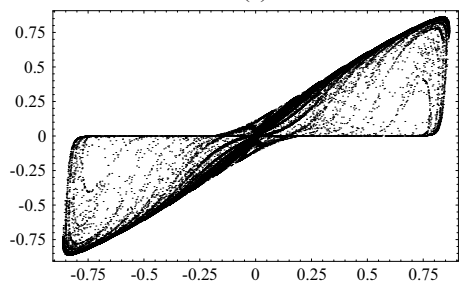

(b)

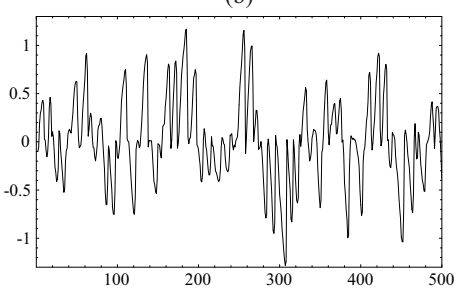

(d)

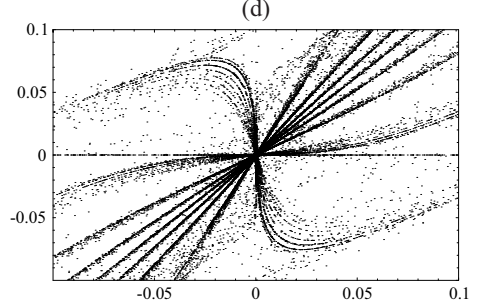

Figure 1.3. Chaotic (top left) and noisy chaotic (top right) time series of asset prices in adaptive belief system with four trader types. Strange attractor (bottom left) and enlargement of strange attractor (bottom right). Belief parameters are: $g_{1}=0, b_{1}=0 ; g_{2}=0.9, b_{2}=0.2$; $g_{3}=0.9, b_{3}=-0.2$ and $g_{4}=1+r=1.01, b_{4}=0$; other parameters are $r=0.01, \beta=90.5$, $w=0$ and $C_{h}=0$ for all $1 \leq h \leq 4$.

For low values of the intensity of choice, the fundamental steady state is stable. As the intensity of choice increases, as in the previous three type example,

\footnotetext{
${ }^{11}$ This result is related to DeLong et al. (1990ab) who show that a constant fraction of noise traders can survive in the market in the presence of fully rational traders. The ABS however are evolutionary models with time varying fractions, driven by strategy performance.
} 

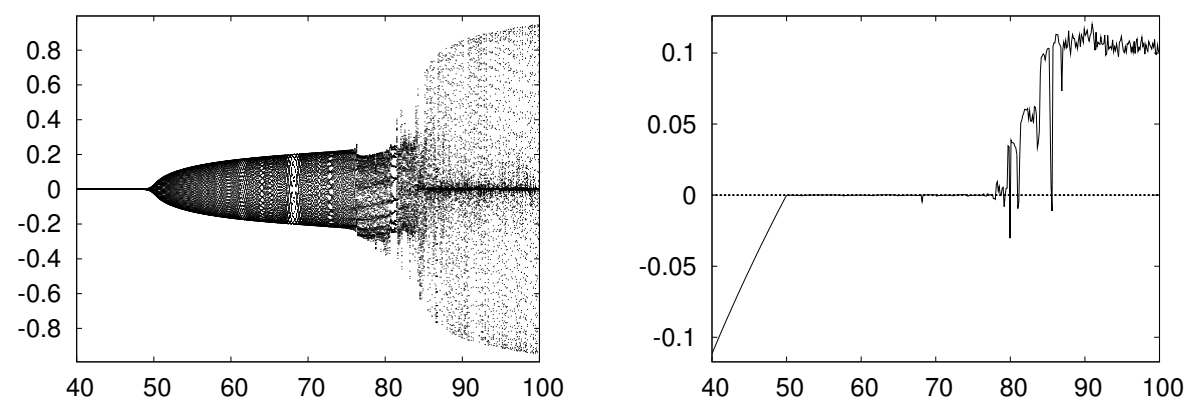

Figure 1.4. Bifurcation diagram and largest Lyapunov exponent plot for 4-type model, buffeted with very small noise $\left(S D=10^{-6}\right.$ for noise term $\epsilon_{t}$ in (1.2.1)), to avoid that for large $\beta$-values the system gets stuck in the locally unstable steady state. Belief parameters are: $g_{1}=0, b_{1}=0 ; g_{2}=0.9, b_{2}=0.2 ; g_{3}=0.9, b_{3}=-0.2$ and $g_{4}=1+r=1.01, b_{4}=0$; other parameters are $r=0.01, \beta=90.5, w=0$ and $C_{h}=0$ for all $1 \leq h \leq 4$. The 4-type model with fundamentalists versus trend followers and biased beliefs exhibits a Hopf bifurcation for $\beta=50$. A rational route to randomness, i.e. a bifurcation route to chaos, occurs, with positive largest Lyapunov exponents, when the intensity of choice becomes large.

the fundamental steady becomes unstable due to a Hopf bifurcation and a stable invariant circle around the unstable fundamental steady state arises, with periodic or quasi-periodic fluctuations. As the intensity of choice further increases, the invariant circle breaks into a strange attractor with chaotic fluctuations. In the evolutionary ABS fundamentalists and chartists co-exist with time varying fractions and prices moving chaotically around the unstable fundamental steady state. Figure 1.4 shows that in this 4-type example with fundamentalists versus trend followers and biased beliefs a rational route to randomness occurs, with positive largest Lyapunov exponents for large values of $\beta$.

This 4-type example shows that, even when there are no information costs for fundamentalists, they cannot drive out other simple trader types and fail to stabilize price fluctuations towards its fundamental value. As in the three type case, the opposite biases create cyclic behavior and, while trend extrapolation turns these cycles into unpredictable chaotic fluctuations.

\subsubsection{Efficiency}

What can be said about market efficiency in an adaptive belief system (ABS)? The (noisy) chaotic price fluctuations are characterized by an irregular switching between phases of close-to-the-fundamental-price fluctuations, phases of 'optimism' with prices following an upward trend, and phases of 'pessimism', with (small) sudden market crashes, as illustrated in Figure 1.3. In fact, in the ABS prices are 
characterized by evolutionary switching between the fundamental value and temporary speculative bubbles. Hence, prices deviate persistently from fundamental value and therefore, prices are excessively volatile and do not reflect economic fundamentals. In this sense the market is inefficient. But are these deviations easy to predict? Even in the simple, stylized 4-type example in the purely deterministic chaotic case, the timing and the direction of the temporary bubbles seem hard to predict, but once a bubble has started, the collapse of the bubble seems to be predictable. In the presence of (small) noise however the situation is quite different, as illustrated in Figure 1.3 (top right): the timing, the direction and the collapse of the bubble all seem hard to predict.

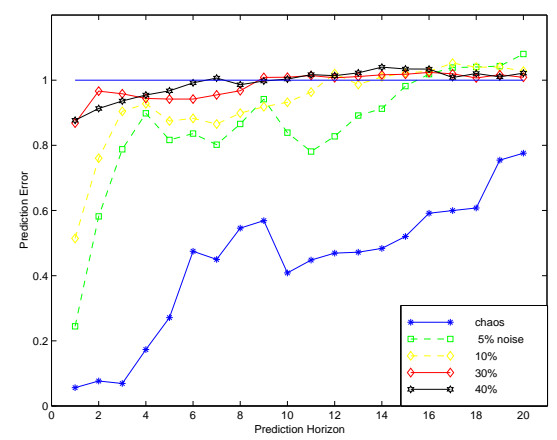

Figure 1.5. Forecasting errors for nearest neighbor method applied to chaotic and noisy chaotic returns series, for different noise levels, in the 4-type adaptive belief system. All returns series have close to zero autocorrelations at all lags. The benchmark case of prediction by the mean 0 is represented by the horizontal line at the normalized prediction error 1 . Nearest neighbor forecasting applied to the purely deterministic chaotic series leads to much smaller forecasting errors at all prediction horizons 1-20 (lowest graph). A noise level of say $10 \%$ means that the ratio of the variance of the noise $\epsilon_{t}$ and the variance of the deterministic price series is $1 / 10$. As the noise level increases, the graphs shift upwards indicating that prediction errors increase. Small dynamic noise thus quickly deteriorates forecasting performance.

To stress this point further, we investigate this (un)predictability, by employing a so called nearest neighbor forecasting method to predict the returns, at lags 1 to 20 for the purely chaotic as well as for several noisy chaotic time series, as illustrated in Figure $1.5^{12}$. Nearest neighbor forecasting looks for patterns in the past that are close to the most recent pattern, and then predicts the average value following all nearby past patterns. According to Takens' embedding theorem this method yields good forecasts for deterministic chaotic systems ${ }^{13}$. Figure 1.5 shows that as the

\footnotetext{
${ }^{12}$ I would like to thank Sebastiano Manzan for providing this figure.

${ }^{13}$ See Kantz and Schreiber (1997) for an extensive treatment of nonlinear time series analysis and forecasting techniques.
} 
noise level increases, the forecasting performance of the nearest neighbor method quickly deteriorates. Hence, in our simple nonlinear evolutionary ABS with noise it is hard to make good forecasts of future returns and to predict when prices will return to fundamental value. Our simple nonlinear ABS with small noise thus captures some of the intrinsic unpredictability of asset returns also present in real markets and in terms of predictability the market is close to being efficient.

\subsubsection{Wealth accumulation}

The evolutionary dynamics in an adaptive belief system (ABS) is driven by realized short run profits, and chartists strategies survive in a world driven by short run profit opportunities. In this subsection, we briefly look at the accumulated wealth in an ABS. Recall that accumulated wealth for strategy type $h$ is given by

$$
W_{h, t+1}=R W_{h t}+\left(p_{t+1}+y_{t+1}-R p_{t}\right) z_{h t}
$$

The first term represents wealth growth due to the risk free asset, while the last term represents wealth growth (or decay) due to investments in the risky asset. Because of market clearing, the average net inflow of wealth due to investment in the risky asset is given by

$$
\sum_{h} n_{h t} z_{h t}\left(p_{t+1}+y_{t+1}-R p_{t}\right)=z^{s}\left(p_{t+1}+y_{t+1}-R p_{t}\right) .
$$

This is the average risk premium required by the population of investors to hold the risky asset. In the special case $z^{s}=0$ the risk premium is 0 and on average wealth of each strategy grows at the risk free rate.

Figure 1.6 shows the development of prices and wealth of each strategy in the 3 -type and 4-type examples of subsections 1.2 .2 and 1.2.3. Prices fluctuate around the fundamental price. For the 3-type example, the wealth accumulated by each of the three strategies, fundamentalists, optimistic biased and pessimistic biased grows over time, at an equal rate. Recall that in the 3 type example, for an infinite intensity of choice $\beta$, the system converges to a stable 4-cycle with average profits equal for all three strategies. At each time $t$, profits of fundamentalists are always between profits of optimists and pessimists, but on average all profits are (almost) equal, and thus accumulated wealth grows at the same rate. ${ }^{14}$

In the 4-type example, trend following strategies are profitable during temporary bubbles. Fundamentalists suffer losses during temporary bubbles, but these losses are limited. When the bubble bursts, fundamentalists make large profits while trend followers suffer from huge losses. On average accumulated wealth of fundamentalists increases, while wealth of chartists decreases, as illustrated in Figure 1.6.

\footnotetext{
${ }^{14}$ For finite intensity of choice, e.g. $\beta=3000$ as in Figure 1.6, wealth of the 3-types grows at almost the same rate. For initial states chosen as in Figure 1.6, wealth of the optimistic types slightly dominates the other two types.
} 

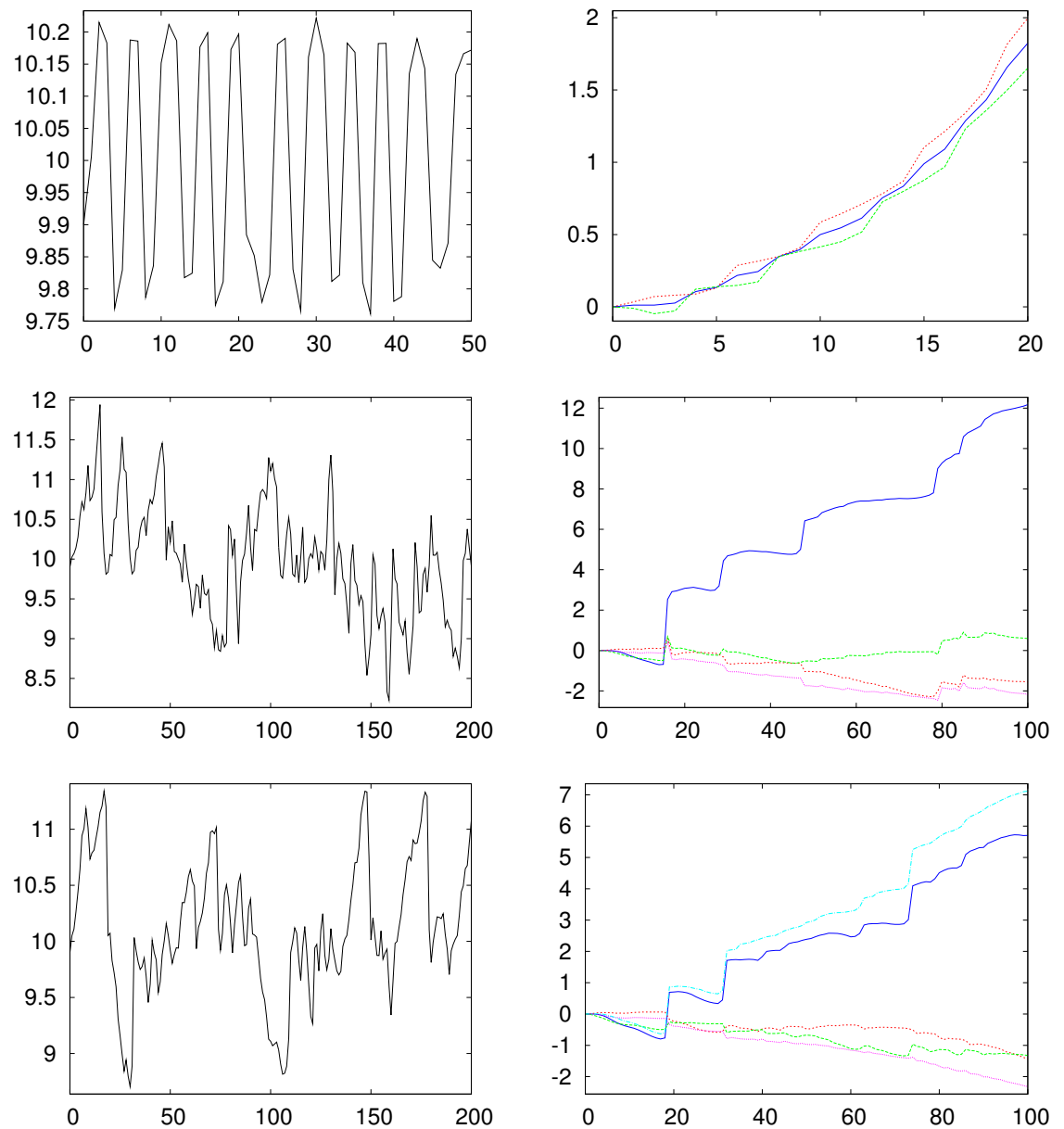

Figure 1.6. Time series of prices (left) and accumulated wealth in 3-type ABS (top panel), 4-type ABS (middle panel) and 5-type ABS (bottom panel). Belief parameters are $b=0.2$ for the 3-type ABS (see subsection 1.2.2) and as in Figure 1.3 in the 4-type case. Other parameters are: $\beta=3000$ and $\sigma_{\epsilon}=0.025$ (3-type ABS); $g_{4}=(1+r)^{2}=1.0201, \beta=180$, $\bar{y}=0.1, R=1.01, \sigma_{\epsilon}=0.2$ (4-type ABS), and $\sigma_{\epsilon}=0.1$ and threshold parameter $\vartheta=0.5$ for switching strategy (5-type ABS). 
The wealth in (1.2.9) corresponds to the accumulated wealth of a trader who always uses strategy $h$. How would a switching strategy perform in a heterogeneous market? Figure 1.6 (bottom panel) illustrates an example of an ABS with 5 strategies, where a switching strategy has been added to the 4-type ABS. The 5th switching strategy is endogenous in the 5-type ABS, and thus affects the realized market price in the same way as the other 4 strategies. The switching strategy always picks the best of the other 4 strategies, according to last period's realized profits, conditional on how far the price deviates from the fundamental benchmark. In the simulation, when the price deviation becomes larger than a threshold parameter $(\vartheta=0.5)$, the switching strategy switches back to the fundamental strategy to avoid losses when the bubble collapses. Figure 1.6 (bottom panel) illustrates two features of the 5-type ABS. Firstly, due to the presence of the switching strategy, the amplitude of price fluctuations (bottom panel, left plot) is somewhat smaller than in the 4-type ABS. This is caused by the switching strategy switching back to the fundamental strategy when the price deviation exceeds the threshold. Secondly, the accumulated wealth of the switching strategy outperforms all other strategies, including the fundamental strategy (Figure 1.6 (bottom panel, right plot). Notice that the two best strategies, the switching strategy and the fundamental strategy, also require most information. The trend following strategies only use publically available information on past prices. ${ }^{15}$ The fundamental strategy uses fundamental information, while the switching strategy uses fundamental information as well as information about competing strategies in the market and their performance.

In the ABS evolutionary framework agents switch strategies based on short run realized profits. In the long run, a fundamental strategy often accumulates more wealth than trend following rules. However, fundamental strategies suffer from losses during temporary bubbles when prices persistently deviate from fundamentals, and may therefore suffer from "limits of arbitrage" (Shleifer and Vishny (1997)). Fundamentalists can stabilize price fluctuations, but only if they are not limited by borrowing constraints or limits of arbitrage. In the long run, a simple switching strategy may accumulate more wealth than a fundamental or technical trading strategy. The fact that a simple switching strategy performs better in a heterogeneous market shows that the ABS-model is behaviorally consistent. Agents have an incentive to keep switching strategies. The switching strategy is very risky however, because it requires good knowledge of the underlying fundamental and good market timing to "get off the bubble before it bursts". Interestingly, Zwart et al. (2007) provided empirical evidence, analyzing 15 emerging market currencies over the period 1995-2006, that a combined strategy with time varying weights may generate economically and statistically significant returns, after accounting for transaction costs. Their strategy is based on a combination of fundamental information on the deviation from purchasing power parity and the real interest rate

\footnotetext{
${ }^{15}$ Recall that these strategies can be formulated without knowledge of the fundamental price, see footnote 4
} 
differential and chartist information from moving average trading rules, with time varying weights determined by relative performance over the past year.

\subsubsection{Extensions}

Several modifications and extensions of a adaptive belief systems (ABS) have been studied. In Brock and Hommes (1998) the demand for the risky asset is derived from a constant absolute risk aversion (CARA) utility function. Chiarella and He (2001) consider the case with constant relative risk aversion (CRRA) utility. This is complicated, because under CARA utility investors' relative wealth affects asset demand and realized asset price, and one has to keep track of the wealth distribution among the population of agents ${ }^{16}$. Anufriev and Bottazzi (2006) and Anufriev (2008) study wealth and asset price dynamics in a heterogeneous agents framework and are able to characterize the type of equilibria and their stability under fairly general behavioral assumptions. Chiarella, Dieci and Gardini $(2002,2006)$ use CRRA utility in an ABS with a market maker price setting rule. Chiarella and $\mathrm{He}$ (2003) and Hommes et al. (2005) investigate an ABS with a market maker price setting rule, and find quite similar dynamical behavior as in the case of a Walrasian market clearing price. De Fontnouvelle (2000) and Goldbaum (2005) apply strategy switching to an asset pricing model with heterogeneous information. Chang (2007) studies how social interactions affect the dynamics of asset prices in an ABS with a Walrasian market clearing price. DeGrauwe and Grimaldi (2005,2006) applied the ABS framework to exchange rate modeling. Chiarella (2009, this handbook) discusses some of these extensions in more detail ${ }^{17}$.

\subsection{MANY TRADER TYPES}

In most heterogeneous agent models (HAMs) in the literature, the number of trader types is small: usally only two, three or four types are considered that use simple fundamentalist or chartist strategies. Generally, analytical tractability can only be obtained at the cost of restricting a HAM to just a few types. Brock, Hommes and Wagener (2005) have however developed a theoretical framework to study evolutionary markets with many different trader types. In this subsection, we discuss their notion of Large Type Limit (LTL), a simple, low dimensional approximation of an evolutionary adaptive belief system (ABS) with many trader types. The LTL can be developed in a fairly general market clearing setting, but here we focus on its application to the asset pricing model with heterogeneous beliefs.

\footnotetext{
${ }^{16}$ In the artifical market of Levy et al. (1994), asset demand is also derived from CRRA utility.

${ }^{17}$ Another related stochastic model with heterogeneous agents and endogenous strategy switching similar to the ABS has been introduced in Föllmer et al. (2005). Scheinkman and Xiong (2004) review related stochastic financial models with heterogeneous beliefs and short sale constraints. Macro models with heterogeneous expectations have been studied, for instance, in Branch and Evans (2006) and Branch and McGough (2008).
} 
Recall from (1.1.11) that in the asset market with $H$ different trader types, the equilibrium price (in deviations $x_{t}$ from the fundamental benchmark) is given by

$$
x_{t}=\frac{1}{1+r} \sum_{h=1}^{H} n_{h t} f_{h t} .
$$

Using the multi-nomial logit probabilities (1.1.13) for the fractions $n_{h t}$ we get

$$
x_{t}=\frac{1}{1+r} \frac{\sum_{h=1}^{H} \mathrm{e}^{\beta U_{h, t-1}} f_{h t}}{\sum_{h=1}^{H} \mathrm{e}^{\beta U_{h, t-1}}} .
$$

It is assumed that prediction and fitness functions take the form $f_{h t}=f\left(\mathbf{x}, \lambda, \vartheta_{h}\right)$ and $U_{h t}=U\left(\mathbf{x}, \lambda, \vartheta_{h}\right)$ respectively, where $\mathbf{x}=\left(x_{t-1}, x_{t-2}, \cdots\right)$ is a vector of lagged deviations from the fundamental, $\lambda$ is a structural parameter vector (e.g. containing the risk free interest rate $r$, the risk aversion parameter $a$, the intensity of choice $\beta$, etc.) and $\vartheta_{h}$ is a multidimensional variable that characterizes the belief type $h$.

The equilibrium equation (1.3.2) determines the evolution of the system with $H$ trader types - this information is coded in the evolution map $\varphi_{H}(\mathbf{x}, \lambda, \vartheta)$ :

$$
\varphi_{H}(\mathbf{x}, \lambda, \vartheta)=\frac{1}{1+r} \frac{\sum_{h=1}^{H} \mathrm{e}^{\beta U\left(\mathbf{x}, \lambda, \vartheta_{h}\right)} f\left(\mathbf{x}, \lambda, \vartheta_{h}\right)}{\sum_{h=1}^{H} \mathrm{e}^{\beta U\left(\mathbf{x}, \lambda, \vartheta_{h}\right)}},
$$

where $\vartheta=\left(\vartheta_{1}, \cdots, \vartheta_{H}\right)$. At the beginning of the market, a large number $H$ of beliefs $\vartheta_{h}$ is sampled from a general distribution of beliefs. For example, all forecasting rules may be drawn from a linear class of rules with $L$ lags,

$$
f_{t}\left(\vartheta_{0}\right)=\vartheta_{00}+\vartheta_{01} x_{t-1}+\vartheta_{02} x_{t-2}+\cdots+\vartheta_{0 L} x_{t-L},
$$

with $\vartheta_{0 h}, h=0, \cdots, L$, drawn from a multivariate normal distribution.

The evolution map $\varphi_{H}$ in (1.3.3) determines the dynamical system corresponding to an asset market with $H$ different belief types. When the number of trader types $H$ is large, this dynamical system contains a large number of stochastic variables $\vartheta=\left(\vartheta_{1}, \ldots, \vartheta_{H}\right)$, where the $\vartheta_{h}$ are IID, with distribution function $F_{\mu}$. At the beginning of the market $H$ belief types are drawn from this distribution, who then compete against each other. The distribution function of the stochastic belief variable $\vartheta_{h}$ depends on a multi-dimensional parameter $\mu$, called the belief parameter. This setup allows to vary the population out of which the individual beliefs are sampled at the beginning of the market.

Observe that both the denominator and the numerator of the evolution map $\varphi_{H}$ in (1.3.3) may be divided by the number of trader types $H$ and thus may be seen as sample means. The evolution map $\psi$ of the large type limit (LTL) is then obtained by replacing sample means in the evolution map $\varphi_{H}$ by population means:

$$
\psi(\mathbf{x}, \lambda, \mu)=\frac{1}{1+r} \frac{E_{\mu}\left[\mathrm{e}^{\beta U\left(\mathbf{x}, \lambda, \vartheta_{0}\right)} f\left(\mathbf{x}, \lambda, \vartheta_{0}\right)\right]}{E_{\mu}\left[\mathrm{e}^{\beta U\left(\mathbf{x}, \lambda, \vartheta_{0}\right)}\right]}=\frac{1}{1+r} \frac{\int \mathrm{e}^{\beta U\left(\mathbf{x}, \lambda, \vartheta_{0}\right)} f\left(\mathbf{x}, \lambda, \vartheta_{0}\right) d \nu_{\mu}}{\int \mathrm{e}^{\beta U\left(\mathbf{x}, \lambda, \vartheta_{0}\right)} d \nu_{\mu}},
$$


where $\vartheta_{0}$ is a stochastic variable, distributed in the same way as the $\vartheta_{h}$, with density $\nu_{\mu}$. The structural parameter vector $\lambda$ of the evolution map $\varphi_{H}$ and of the LTL evolution map $\psi$ coincide. However, whereas the evolution map $\varphi_{H}$ in (1.3.3) of the heterogeneous agent system contains $H$ randomly drawn multi-dimensional stochastic variables $\vartheta_{h}$, the LTL evolution map $\psi$ in (1.3.5) only contains the belief parameter vector $\mu$ describing the joint probability distribution. Taking a large type limit thus leads to a huge reduction in stochastic belief variables.

According to the LTL-theorem of Brock et al. (2005), as the number $H$ of trader types tends to infinity, the $H$-type evolution map $\varphi$ converges almost surely to the LTL-map $\psi$. This implies that the corresponding LTL dynamical system is a good approximation of the dynamical behavior in a heterogeneous asset market when the number of belief types $H$ is large. In particular, all generic and persistent dynamic properties will be preserved with high probability. For example, if the LTL-map exhibits a bifurcation route to chaos for one of the structural parameters, then, if the number of trader types $H$ is large, the $H$-type system also exhibits such a bifurcation route to chaos with high probability.

A straightforward computation using moment generating functions shows that, for example, in the case of linear forecasting rules (1.3.4) with three lags $(L=3)$, the corresponding LTL becomes a 5 -D nonlinear system given by

$$
\begin{aligned}
& (1+r) x_{t}=\mu_{0}+\mu_{1} x_{t-1}+\mu_{2} x_{t-2}+\mu_{3} x_{t-3} \\
& \quad+\eta\left(x_{t-1}-R x_{t-2}+a \sigma^{2} z^{s}\right)\left(\sigma_{0}^{2}+\sigma_{1}^{2} x_{t-1} x_{t-3}+\sigma_{2}^{2} x_{t-2} x_{t-4}+\sigma_{3}^{2} x_{t-3} x_{t-5}\right)
\end{aligned}
$$

where $\eta=\beta /\left(a \sigma^{2}\right)$. The simplest special case of (1.3.6) that still leads to interesting dynamics is obtained when all $\vartheta_{0 k}=0,1 \leq k \leq d$, that is, when the forecasting function (1.3.4) is purely biased: $f_{t}\left(\vartheta_{0}\right)=\vartheta_{00}$. The LTL then simplifies to the linear system

$$
R x_{t}=\mu_{0}+\eta \sigma_{0}^{2}\left(x_{t-1}-R x_{t-2}+a \sigma^{2} z^{s}\right) .
$$

This simplest case already provides insight into the (in)stability of the (fundamental) steady state in an evolutionary system with many trader types. When there is no intrinsic mean bias, that is when the mean of the biases $\vartheta_{00}$ equals 0 (i.e. $\mu_{0}=0$ ), and the risk premium is zero $\left(z^{s}=0\right)$, the steady state of the LTL (1.3.7) coincides with the fundamental: $x^{*}=0$. When the mean bias and risk premium are both positive (negative) the steady state deviation $x^{*}$ will be positive (negative) so that the steady state will be above (below) the fundamental. The natural bifurcation parameter tuning the (in)stability of the system is $\eta \sigma_{0}^{2}=\beta \sigma_{0}^{2} / a \sigma^{2}$. We see that instability occurs if and only if $\eta$ increases beyond the bifurcation point $\eta_{c}=1 / \sigma_{0}^{2}$. Hence this simple case already suggests forces that may destabilize the evolutionary system: an increase in choice intensity $\beta$ for evolutionary selection, a decrease in risk aversion $a$, a decrease in conditional variance of excess returns $\sigma^{2}$, or an increase in the diversity of purely biased beliefs $\sigma_{0}^{2}$. All of these forces can push $\eta$ beyond $\eta_{c}$, thereby triggering instability of the (fundamental) steady state. 
For the large type limit (LTL) in (1.3.6), in the case of linear forecasting rules with 3 lags, a bifurcation route to chaos, with asset prices fluctuating around the unstable fundamental steady state, occurs when $\eta$ increases. This shows that a rational route to randomness can occur in an asset market with many different trader types, when traders become increasingly sensitive to differences in fitness (i.e. an increase in the intensity of choice $\beta$ ) or traders become less risk averse (i.e. a decrease of the coefficient of risk aversion $a$ ). In a many trader types evolutionary world fundamentalists will in general not drive out all other types and asset prices need not converge to their fundamental value.

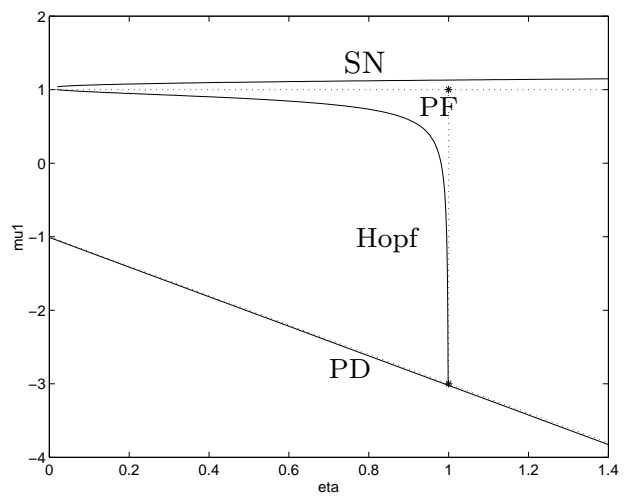

Figure 1.7. Bifurcation diagram in the $\left(\eta, \mu_{1}\right)$ parameter plane for the large type limit (LTL) (1.3.6), where $\mu_{1}$ represents the mean of the first order stochastic trend variable $\vartheta_{01}$ in the forecasting rule (1.3.4). For $\mu_{0}=a \sigma^{2}=0$, with $\mu_{0}$ the mean of the constant $\vartheta_{00}$ in the forecasting rule (1.3.4), the LTL is symmetric and thus non-generic (dotted curves); when $\mu_{0} \neq 0$ the LTL is non-symmetric and generic. The diagrams show Hopf $(H)$, period doubling $(P D)$, pitchfork $(P F)$ and saddle-node $(S N)$ bifurcation curves in $\left(\eta, \mu_{1}\right)$ parameter plane, with other parameters fixed at $R=1.01, z^{s}=0, \mu_{2}=\mu_{3}=0$, $\sigma_{0}=\sigma_{1}=\sigma_{2}=1$ and $\sigma_{3}=0$. Between the Hopf and PD curves (and the PF curve when $\left.\mu_{0}=0\right)$ there is a unique, stable steady state. This steady state becomes unstable when crossing the Hopf or the PD curve. Above the PF curve or the SN curve the system has three steady states. The PF curve is non-generic and only arises in the symmetric case with mean bias $\mu_{0}=0$. When the symmetry is broken by perturbing the mean bias to $\mu_{0}=-0.1$, the PF curve 'breaks' into generic Hopf and SN curves.

Figure 1.7 shows a 2-dimensional bifurcation diagram in the $\left(\eta, \mu_{1}\right)$ parameter plane, where $\mu_{1}$ represents the mean of the first order stochastic trend variable $\vartheta_{01}$ in the forecasting rule (1.3.4). Recall that $\mu_{0}$ is the mean of the constant term $\vartheta_{00}$ in the forecasting rule (1.3.4); it models the 'mean bias' of the trader type. When $\mu_{0}=0$ and $a \sigma^{2} z_{s}=0$ (expressing that the risk premium is zero), the LTL is symmetric with respect to the fundamental steady state. 
In the symmetric case ( dotted lines in figure 1.7), for parameters taking values in the region enclosed by the Hopf, period doubling (PD) and pitchfork (PF) bifurcation curves, the fundamental steady state is unique and stable ${ }^{18}$. As the parameters cross the PF curve, two additional non-fundamental steady states are created, one above and one below the fundamental. Another route to instability occurs when crossing the Hopf curve, where the fundamental steady state becomes unstable and a (stable) invariant circle with periodic or quasi-periodic dynamics is created. The pitchfork bifurcation curve is non-generic, and only occurs in the symmetric case. When the symmetry is broken by a non-zero mean bias $\mu_{0} \neq 0$, as illustrated in figure 1.7 (bold curves) for $\mu_{0}=-0.1$, the PF curve disappears, and "breaks" into two generic co-dimension one bifurcation curves, a Hopf and a saddle-node (SN) bifurcation curve. When crossing the SN curve from below, two additional steady states are created, one stable and one unstable. Notice that, as illustrated in figure 1.7, when the perturbation is small (in the figure $\mu_{0}=-0.1$ ), the SN and the Hopf curves are close to the PF and the Hopf curves (dotted lines) in the symmetric case. In this sense the bifurcation diagram depends continuously on the parameters, and it is useful to consider the symmetric LTL as an "organizing" center to study bifurcation phenomena in the generic, non-symmetric LTL.

The most relevant case from an economic viewpoint arises when the mean $\mu_{1}$ of the first order coefficient $\vartheta_{01}$ in the forecasting rule (1.3.4) satisfies $0 \leq \mu_{1} \leq 1$. In that case, the (fundamental) steady state loses stability in a Hopf bifurcation as $\eta$ increases. Figure 1.8 illustrates the dynamical behavior of the LTL as the parameter $\eta$ further increases. After the Hopf bifurcation periodic and quasi-periodic dynamics on a stable invariant circle occur, and for increasing values of $\eta$ a bifurcation route to strange attractors occurs. Figure 1.8 thus presents numerical evidence of the occurrence of a rational route to randomness, that is, a bifurcation route to strange attractors as the intensity of choice to switch forecasting strategies increases. If such rational routes to randomness occur for the LTL, the LTL convergence theorem implies that in evolutionary systems with many trader types rational routes to randomness occur with high probability.

Diks and van der Weide $(2003,2005)$ have generalized the notion of LTL and introduced so-called Continuous Belief Systems $(C B S)$, where the beliefs of traders are distributed according to a continuous density function. The beliefs distribution function and the equilibrium prices co-evolve over time. The LTL theory discussed here as well as its extensions can be used to form a bridge between an analytical approach and the literature on evolutionary artificial market simulation models reviewed in LeBaron $(2000,2006)$.

\footnotetext{
${ }^{18}$ See the appendix for a brief introduction to bifurcation theory, illustrated by simple examples of the saddle-node, period doubling, pitchfork and Hopf bifurcations.
} 


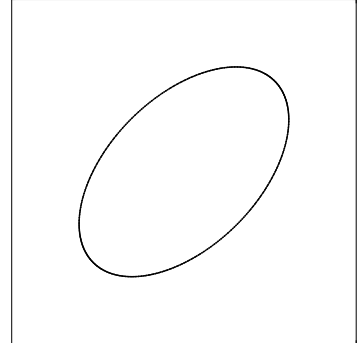

(a) $\eta=1.4$

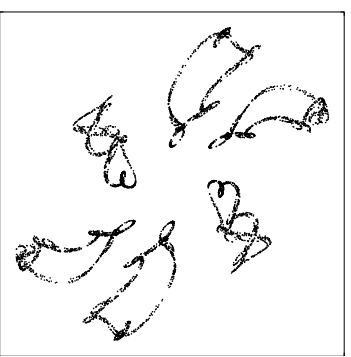

(d) $\eta=1.57$

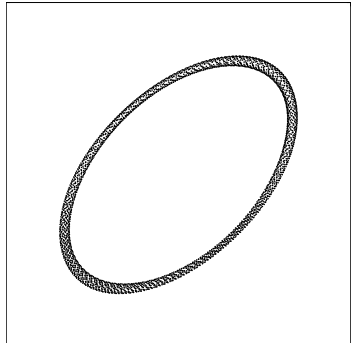

(b) $\eta=1.51$

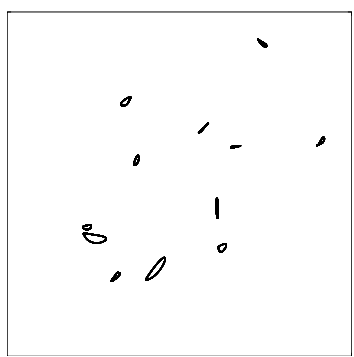

(e) $\eta=1.59$

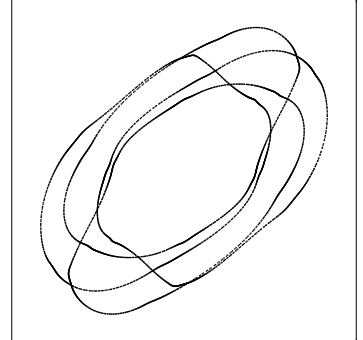

(c) $\eta=1.52$

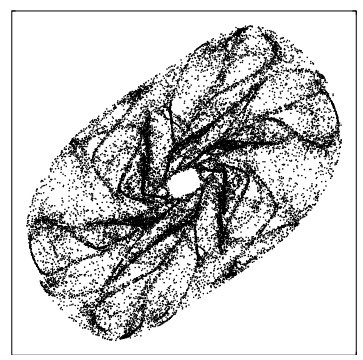

(f) $\eta=1.6$

Figure 1.8. Attractors in the phase space for the 5-D LTL with parameters $R=1.01$, $z^{s}=0, \mu_{0}=0, \mu_{1}=0, \mu_{2}=\mu_{3}=0, \sigma_{0}=\sigma_{1}=\sigma_{2}=\sigma_{3}=1:$ (a) immediately after the Hopf bifurcation (quasi-)periodic dynamics on a stable invariant circle occurs; (b-c) after a Hopf bifurcation (quasi-)periodic dynamics on a stable invariant torus occurs; $(d-f)$ breaking up of the invariant torus into a strange attractor.

\subsection{EMPIRICAL VALIDATION}

In this section we discuss the empirical validity of the asset pricing model with heterogeneous beliefs. There is already a large literature on heterogeneous agent models (HAMs) replicating many of the important stylized facts of financial time series on short time scales (say daily or higher frequency), such as fat tails and long memory in the returns distribution and clustered volatility. Examples of heterogeneous agent models able to replicate stylized facts of financial markets include e.g. Brock and LeBaron (1996), Arthur et al. (1997), Brock and Hommes (1997b), Youssefmir and Huberman (1997), LeBaron et al. (1999), Lux and Marchesi (1999,2000), Farmer and Joshi (2002), Kirman and Teyssière (2002), Hommes (2002), Iori (2002), Cont and Bouchaud (2000) and Gaunersdorfer and Hommes (2007). The recent survey by Lux (2009, this Handbook) contains an extensive survey of behavioral interacting agent models mimicking the stylized facts of asset returns. We have already seen examples of simple heterogeneous agent models 
mimicking temporary bubbles and crashes. In this section we discuss how these qualitative features match with observed bubbles and crashes in real markets.

Empirical validation and estimation of HAMs on economic or financial data is still in its infancy. An early attempt has already been made by Shiller (1984), who presents a HAM with smart money traders, having rational expectations, versus ordinary investors (whose behavior is in fact not modeled at all). Shiller estimates the fraction of smart money investors over the period 1900-1983, and finds considerable fluctuations of the fraction over a range between 0 and 50\%. Baak (1999) and Chavas (2000) estimate HAMs on hog and beef market data, and found evidence for the heterogeneity of expectations. Winker and Gilli (2001) and Gilli and Winker (2003) estimate the model of Kirman $(1991,1993)$ with fundamentalists and chartists, using the daily DM-US\$ exchange rates 1991-2000. Their estimated parameter values correspond to a bimodal distribution of agents. Westerhoff and Reitz (2003) also estimate an HAM with fundamentalists and chartists to exchange rates and find considerable fluctuations of the market impact of fundamentalists. Alfarano et al. (2005) estimate an agent-based herding model where agents switch between fundamentalist and chartist strategies. Branch (2004) estimates a model with heterogeneous beliefs and time varying fractions, using survey data on inflation expectations. In this section, we discuss the estimation of a simple two type asset pricing model with heterogeneous beliefs, as discussed in section 1.1, on yearly S\&P 500 data, 1871-2003, as done in Boswijk et al. (2007). As we will see, this simple 2-type model can e.g. explain the dot com bubble in the late 1990s and the subsequent crash early $2000^{19}$.

\subsubsection{The model in price-to-cash flows}

In the previous section, the dividend process of the risky asset has been assumed to be stationary. To estimate the model using yearly data of more than a century, the dividend process has to be taken growing over time and thus non-stationary. In order to estimate a simple 2-type model, Boswijk et al. (2007) therefore reformulated the model in terms of price-to-cash flows. Recall from (1.1.5) that, under the assumption of zero net supply of the risky asset, the equilibrium pricing equation is

$$
p_{t}=\frac{1}{1+r} \sum_{h=1}^{H} n_{h, t} E_{h, t}\left(p_{t+1}+y_{t+1}\right),
$$

\footnotetext{
${ }^{19}$ Van Norden and Schaller (1999) estimate a nonlinear time series switching model with two regimes, an explosive and a collapsing bubble regime, with the probability of being in the explosive regime depends negatively on the relative absolute deviation of the bubble from the fundamental. Brooks and Katsaris (2005) extend this model to three regimes, adding a third dormant bubble regime where the bubble grows at the required rate of return without explosive expectations.
} 
or equivalently

$$
r=\sum_{h=1}^{H} n_{h, t} \frac{E_{h, t}\left[p_{t+1}+y_{t+1}-p_{t}\right]}{p_{t}} .
$$

In equilibrium the average required rate of return for investors to hold the risky asset equals the discount rate $r$. In the estimation of the model the discount rate $r$ has been set equal to the sum of the (risk free) interest rate and the required risk premium on stocks. A simple, non-stationary process that fits cash flow data (dividends or earnings) well is a stochastic process with a constant growth rate. More precisely, assume that $\log y_{t}$ is a Gaussian random walk with drift, that is,

$$
\log y_{t+1}=\mu+\log y_{t}+v_{t+1}, \quad v_{t+1} \sim \text { i.i.d. } N\left(0, \sigma_{v}^{2}\right)
$$

which implies

$$
\frac{y_{t+1}}{y_{t}}=e^{\mu+v_{t+1}}=e^{\mu+\frac{1}{2} \sigma_{v}^{2}} e^{v_{t+1}-\frac{1}{2} \sigma_{v}^{2}}=(1+g) \varepsilon_{t+1},
$$

where $g=e^{\mu+\frac{1}{2} \sigma_{v}^{2}}-1$ and $\varepsilon_{t+1}=e^{v_{t+1}+\frac{1}{2} \sigma_{v}^{2}}$, so that $E_{t}\left(\varepsilon_{t+1}\right)=1$. As before, we assume that all types have correct beliefs on the cash flow, that is,

$$
E_{h, t}\left[y_{t+1}\right]=E_{t}\left[y_{t+1}\right]=(1+g) y_{t} E_{t}\left[\varepsilon_{t+1}\right]=(1+g) y_{t}
$$

Since the cash flow is an exogenously given stochastic process it seems natural to assume that agents have learned the correct beliefs on next period's cash flow $y_{t+1}$. In particular, boundedly rational agents can learn about the constant growth rate e.g. by running a simple regression of $\log \left(y_{t} / y_{t-1}\right)$ on a constant. In contrast, prices are determined endogenously and are affected by expectations about next period's price. In a heterogeneous world, agreement about future prices therefore seems more unlikely than agreement about future cash flows. Therefore we assume homogeneous beliefs about future cash flow, but heterogeneous beliefs about future prices. $^{20}$ The pricing equation (1.4.1) can be reformulated in terms of price-tocash-flow $(\mathrm{P} / \mathrm{Y})$ ratio, $\delta_{t}=p_{t} / y_{t}$, as ${ }^{21}$

$$
\delta_{t}=\frac{1}{R^{*}}\left\{1+\sum_{h=1}^{H} n_{h, t} E_{h, t}\left[\delta_{t+1}\right]\right\}, \quad R^{*}=\frac{1+r}{1+g} .
$$

In the special case when all agents have rational expectations the equilibrium pricing equation (1.4.1) simplifies to $p_{t}=(1 /(1+r)) E_{t}\left(p_{t+1}+y_{t+1}\right)$. It is well known

\footnotetext{
${ }^{20}$ Barberis et al. (1998) consider a model where agents are affected by psychological biases in forming expectations about future cash flows. In particular, agents may overreact to good news about economic fundamentals because they believe that cash flows have moved into another regime with higher growth. Their model is able to explain continuation and reversal of stock returns.

${ }^{21}$ In what follows we will use either price-to-dividend $(\mathrm{P} / \mathrm{D})$ or price-to-earnings $(\mathrm{P} / \mathrm{E}) \mathrm{ratio}$, and use the general notation $\mathrm{P} / \mathrm{Y}$ for price-to-cash-flows.
} 
that, in the case of a constant discount rate $r$ and a constant growth rate $g$ for dividends, according to the static Gordon growth model (Gordon, 1962), the rational expectations fundamental price, $p_{t}^{*}$, of the risky asset is given by

$$
p_{t}^{*}=\frac{1+g}{r-g} y_{t}, \quad \quad r>g .
$$

Equivalently, in terms of price-to-cash flow ratios the fundamental is

$$
\delta_{t}^{*}=\frac{p_{t}^{*}}{y_{t}}=\frac{1+g}{r-g} \equiv m .
$$

We will refer to $p_{t}^{*}$ as the fundamental price and to $\delta_{t}^{*}$ as the fundamental $\mathrm{P} / \mathrm{Y}$-ratio. When all agents are rational the pricing equation (1.4.6) in terms of the $\mathrm{P} / \mathrm{Y}$-ratio, $\delta_{t}=p_{t} / y_{t}$, becomes

$$
\delta_{t}=\frac{1}{R^{*}}\left\{1+E_{t}\left[\delta_{t+1}\right]\right\} .
$$

In terms of the deviation from the fundamental ratio, $x_{t}=\delta_{t}-\delta_{t}^{*}=\delta_{t}-m$, this simplifies to

$$
x_{t}=\frac{1}{R^{*}} E_{t}\left[x_{t+1}\right] .
$$

Under heterogeneity in expectations, the pricing equation (1.4.6) is expressed in terms of $x_{t}$ as

$$
x_{t}=\frac{1}{R^{*}} \sum_{h=1}^{H} n_{h, t} E_{h, t}\left[x_{t+1}\right] .
$$

\section{Heterogeneous beliefs}

The expectation of belief type $h$ about next period $\mathrm{P} / \mathrm{Y}$-ratio is expressed as

$$
E_{h, t}\left[\delta_{t+1}\right]=E_{t}\left[\delta_{t+1}^{*}\right]+f_{h}\left(x_{t-1}, \ldots, x_{t-L}\right)=m+f_{h}\left(x_{t-1}, \ldots, x_{t-L}\right),
$$

where $\delta_{t}^{*}$ represents the fundamental price-to-cash-flow ratio $\mathrm{P} / \mathrm{Y}, E_{t}\left(\delta_{t+1}^{*}\right)=m$ is the rational expectation of the $\mathrm{P} / \mathrm{Y}$-ratio available to all agents, $x_{t}$ is the deviation of the $\mathrm{P} / \mathrm{Y}$-ratio from its fundamental value and $f_{h}(\cdot)$ represents the expected transitory deviation of the $\mathrm{P} / \mathrm{Y}$-ratio from the fundamental value, depending on $L$ past deviations. The information available to investors at time $t$ includes present and past cash flows and past prices. In terms of deviations from the fundamental $\mathrm{P} / \mathrm{Y}$-ratio, $x_{t}$, we get

$$
E_{h, t}\left[x_{t+1}\right]=f_{h}\left(x_{t-1}, \ldots, x_{t-L}\right) .
$$

Note again that the rational expectations, fundamental benchmark is nested in the heterogeneous agent model as a special case when $f_{h} \equiv 0$ for all types $h$. We can express Equation (1.4.11) as

$$
R^{*} x_{t}=\sum_{h=1}^{H} n_{h, t} f_{h}\left(x_{t-1}, \ldots, x_{t-L}\right) .
$$


From this equilibrium equation it is clear that the adjustment towards the fundamental P/Y-ratio will be slow if a majority of investors has persistent beliefs about it.

\section{Evolutionary selection of expectations}

In addition to the empirical evidence of persistent deviations from fundamentals there is also significant evidence of time variation in the sentiment of investors. This has been documented, for example, by Shiller $(1987,2000)$ using survey data. In the model considered here, agents are boundedly rational and switch between different forecasting strategies according to recently realized profits. We denote by $\pi_{h, t-1}$ the realized profits of type $h$ at the end of period $t-1$, given by (cf. 1.1.14)

$$
\pi_{h, t-1}=R_{t-1} z_{h, t-2}=R_{t-1} \frac{E_{h, t-2}\left[R_{t-1}\right]}{a V_{t-2}\left[R_{t-1}\right]},
$$

where $R_{t-1}=p_{t-1}+y_{t-1}-(1+r) p_{t-2}$ is the realized excess return at time $t-1$ and $z_{h, t-2}$ is the demand of the risky asset by belief type $h$, as given in (1.1.3), formed in period $t-2$. As before, we assume that the beliefs about the conditional variance of excess returns are the same for all types and equal to fundamentalists beliefs about conditional variance, i.e.

$$
V_{h, t-2}\left[R_{t-1}\right]=V_{t-2}\left[P_{t-1}^{*}+y_{t-1}-(1+r) P_{t-2}^{*}\right]=y_{t-2}^{2} \eta^{2},
$$

where $\eta^{2}=(1+m)^{2}(1+g)^{2} V_{t-2}\left[\epsilon_{t-1}\right]$, with $\epsilon_{t}$ IID noise driving the cash flow. The fitness measure can be rewritten in terms of the deviation $x_{t}=\delta_{t}-m$ of the $\mathrm{P} / \mathrm{Y}$ ratio from its fundamental value, with $m=(1+g) /(r-g)$ as

$$
\pi_{h, t-1}=\frac{(1+g)^{2}}{a \eta^{2}}\left(x_{t-1}-R^{*} x_{t-2}\right)\left(E_{h, t-2}\left[x_{t-1}\right]-R^{*} x_{t-2}\right) .
$$

This fitness measure has a simple, intuitive explanation in terms of forecasting performance for next period's deviation from the fundamental. A positive demand $z_{h, t-2}$ may be seen as a bet that $x_{t-1}$ would go up more than what was expected on average from $R^{*} x_{t-2}$ (note that $R^{*}$ is the growth rate of rational bubble solutions). The realized fitness $\pi_{h, t-1}$ of strategy $h$ is the realized profit from that bet and it will be positive if both the realized deviation $x_{t-1}>R^{*} x_{t-2}$ and the forecast of the deviation $E_{h, t-2}\left[x_{t-1}\right]>R^{*} x_{t-2}$. More generally, if both the realized absolute deviation $\left|x_{t-1}\right|$ and the absolute predicted deviation $\left|E_{h, t-2}\left[x_{t-1}\right]\right|$ to the fundamental value are larger than $R^{*}$ times the absolute deviation $\left|x_{t-2}\right|$, then strategy $h$ generates positive realized fitness. In contrast, a strategy that wrongly predicts whether the asset price mean reverts back towards the fundamental value or moves away from the fundamental generates a negative realized fitness.

At the beginning of period $t$ investors compare the realized relative performances of the different strategies and withdraw capital from those that performed poorly 
and move it to better strategies. The fractions $n_{h, t}$ evolve according to a discrete choice model with multi-nomial logit probabilities, that is (cf. (1.1.13)),

$$
n_{h, t}=\frac{\exp \left[\beta \pi_{h, t-1}\right]}{\sum_{k=1}^{H} \exp \left[\beta \pi_{k, t-1}\right]}=\frac{1}{1+\sum_{k \neq h} \exp \left[-\beta \Delta \pi_{t-1}^{h, k}\right]},
$$

where $\beta>0$ is the intensity of choice as before, and $\Delta \pi_{t-1}^{h, k}=\pi_{h, t-1}-\pi_{k, t-1}$ denotes the difference in realized profits of belief type $h$ compared to type $k$.

\subsubsection{Estimation of a simple two-type example}

Consider the case of two types, both predicting next period's deviation by extrapolating past realizations in a linear fashion, that is ${ }^{22}$

$$
E_{h, t}\left[x_{t+1}\right]=f_{h}\left(x_{t-1}\right)=\varphi_{h} x_{t-1} .
$$

The dynamic asset pricing model with two types can then be written as

$$
R^{*} x_{t}=n_{t} \varphi_{1} x_{t-1}+\left(1-n_{t}\right) \varphi_{2} x_{t-1}+\epsilon_{t},
$$

where $\varphi_{1}$ and $\varphi_{2}$ denote the coefficients of the two belief types, $n_{t}$ represents the fraction of investors that belong to the first type of traders and $\epsilon_{t}$ represents a disturbance term. The value of the parameter $\varphi_{h}$ can be interpreted as follows. If it is positive and smaller than 1 , investors expect the stock price to mean revert towards the fundamental value. This type of agents represent fundamentalists, because they expect the asset price to move back towards its fundamental value in the long run. The closer $\varphi_{h}$ is to 1 the more persistent are the expected deviations. If the beliefs parameter $\varphi_{h}$ is larger than 1, it implies that investors believe the deviation of the stock prices to grow over time at a constant speed. We will refer to this type of agents as trend followers. Note in particular that when one group of investors believes in a strong trend, i.e. $\varphi_{h}>R^{*}$, this may cause asset prices to deviate further from their fundamental value. In the case with 2 types with linear beliefs (1.4.19), the fraction of type 1 investors is

$$
n_{t}=\frac{1}{1+\exp \left\{-\beta^{*}\left[\left(\varphi_{1}-\varphi_{2}\right) x_{t-3}\left(x_{t-1}-R^{*} x_{t-2}\right)\right]\right\}}
$$

where $\beta^{*}=\beta(1+g)^{2} /\left(a \eta^{2}\right)$.

The two-type model (1.4.20) and (1.4.21) has been estimated, using an updated version of the data set described in Shiller (1989), consisting of annual observations of the S\&P500 index from 1871 to 2003 . Here we present the estimation results

\footnotetext{
${ }^{22}$ In the estimation of the model higher order lags turned out to be insignificant, so we focus on the simplest case with only one lag in the function $f_{h}(\cdot)$, with $\varphi_{h}$ the parameter characterizing the strategy of type $h$.
} 
with earnings as cash flows, but using dividends as cash flows gives similar results. The valuation ratios are then the Price-to-Earnings (PE) ratios. ${ }^{23}$

Recall that according to the static Gordon growth model the fundamental price is given by

$$
p_{t}^{*}=m y_{t}, \quad m=\frac{1+g}{r-g} .
$$

The fundamental value of the asset is a multiple $m$ of its cash flow where $m$ depends on the discount rate $r$ and the cash flow growth rate $g$. The multiple $m$ can also be interpreted as the price-to-dividend $(\mathrm{P} / \mathrm{D})$ or the price-to-earnings $(\mathrm{P} / \mathrm{E})$ ratios implied by the present value model. Figure 1.9 shows the $(\log )$ of yearly S\&P 500 data together with the fundamental benchmark as well as their P/E-ratio's. The figure shows a clear long-term co-movement of the stock price and the fundamental value. However, the $\mathrm{P} / \mathrm{E}$-ratio takes persistent swings away from the constant value predicted by the present value model. This suggests that the fundamental value does not account completely for the dynamics of stock prices, as was suggested in the early debate on mean reversion by Summers (1986). A survey of the on-going debate is given in Campbell and Shiller (2005). Here we use the simple constant growth Gordon model for the fundamental price and estimate the two type model on deviations from this benchmark. ${ }^{24}$

Recall that $R^{*}=(1+r) /(1+g)$, where $g$ is the constant growth rate of the cash flow and $r$ is the discount rate $r$ equal to the risk free interest rate plus a risk premium. We use an estimate of the risk premium -the difference between the expected return on the market portfolio of common stocks and the risk-free interest rate- to obtain $R^{*}$, as in Fama and French (2002). The risk premium safisfies

$$
R P=g+y / p-i
$$

where $g$ is the growth rate of dividends, $y / p$ denotes the average dividend yield $y_{t} / p_{t-1}$ and $i$ is the risk free interest rate. For annual data from 1871 to 2003 of the S\&P500 the estimates are $i=2.57 \%$ and $R P=6.56 \%$, so that $r=9.13 \%$ and $R^{*}=1.074 .^{25}$ The corresponding average price-to-earnings ratio is 13.4 , as illustrated in Figure 1.9.

Using yearly data of the S\&P 500 index from 1871 to 2003 , the parameters $\left(\varphi_{1}^{\prime}, \varphi_{2}^{\prime}, \beta^{*}\right)$ in the 2 -type model $(1.4 .20)$ and (1.4.21) can be estimated by nonlinear least squares. The estimation results are as follows:

\footnotetext{
${ }^{23}$ Since earnings data are noisy, to determine the fundamental valuation we follow the practice of Campbell and Shiller (2005) to smooth earnings by a 10 years moving average.

${ }^{24}$ The same approach can be used for more general, time varying fundamental processes. Manzan (2003) shows that a dynamic Gordon model for the fundamental price, where the discount rate $r$ and/or the growth rate $g$ are time varying does not explain the large fluctuations in price-tocash-flow ratios, and in fact yields a fundamental price pattern close to that for the static Gordon model. Boswijk et al. (2007) also estimate a version of the model allowing for time variation in the growth rate of the cash flow, and obtain similar results.

${ }^{25}$ These estimates are slightly different from Fama and French (2002), because as in Shiller (1989), we use the CPI index to deflate nominal values.
} 


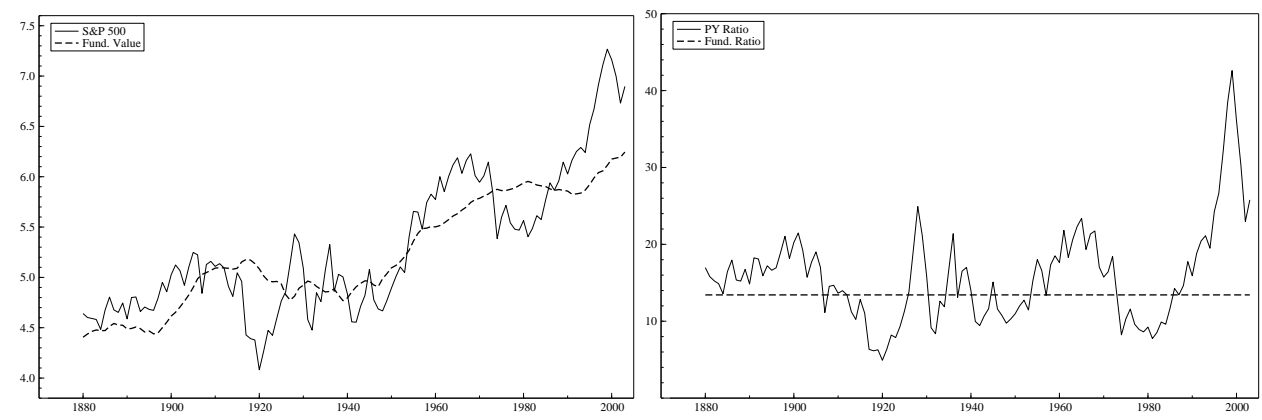

Figure 1.9. Yearly S\&P 500, $1871-2003$ and benchmark fundamental $p_{t}^{*}=m y_{t}$, with $m=(1+g) /(1+r)$. The left panel shows logs of S\&P 500 and the log of the fundamental $p_{t}^{*}$, while the right panel shows the $\mathrm{P} /$ E-ratio of the S\&P 500 around the constant fundamental benchmark $p_{t}^{*} / y_{t}=m$.

$$
\begin{gathered}
R^{*} x_{t}=n_{t} \begin{cases}0.80 & \left.x_{t-1}\right\} \\
(0.074) & \left(1-n_{t}\right)\left\{1.097 x_{t-1}\right\}+\widehat{\epsilon}_{t} \\
n_{t}=\{1+\exp [-7.54(-0.052) \\
\end{cases}
\end{gathered}
$$

$R^{2}=0.77, A I C=2.23, A I C_{A R(1)}=2.29, \quad \varphi_{A R(1)}=0.983, Q_{L B}(4)=0.94, F^{b o o t}(p$-value $)=10.15(0.011)$

The belief coefficients are strongly significant and different from each other. On the other hand, the intensity of choice $\beta^{*}$ is not significantly different from zero. This is a common result in nonlinear switching-type regression models, where the parameter $\beta^{*}$ in the transition function is difficult to estimate and has a large standard deviation, because relatively large changes in $\beta^{*}$ cause only small variation of the fraction $n_{t}$. Teräsvirta (1994) argues that this should not be worrying as long as there is significant heterogeneity in the estimated regimes. The nonlinear switching model achieves a lower value for the AIC selection criterion compared to a linear AR(1) model. This suggests that the model is capturing nonlinearity in the data. This is also confirmed by the bootstrap F-test for linearity, which strongly rejects the null hypothesis of linearity in favor of the heterogeneous agent model. The residuals of the regression do not show significant evidence of autocorrelation at the $5 \%$ significance level.

The estimated coefficient of the first regime is 0.80 , corresponding to an halflife of about three years. The first regime can be characterized as fundamentalist beliefs, expecting the asset price to move back towards its fundamental value. In contrast, the second regime has an estimated coefficient close to 1.1, implying that in this regime agents are trend followers, believing the deviation of the stock price 

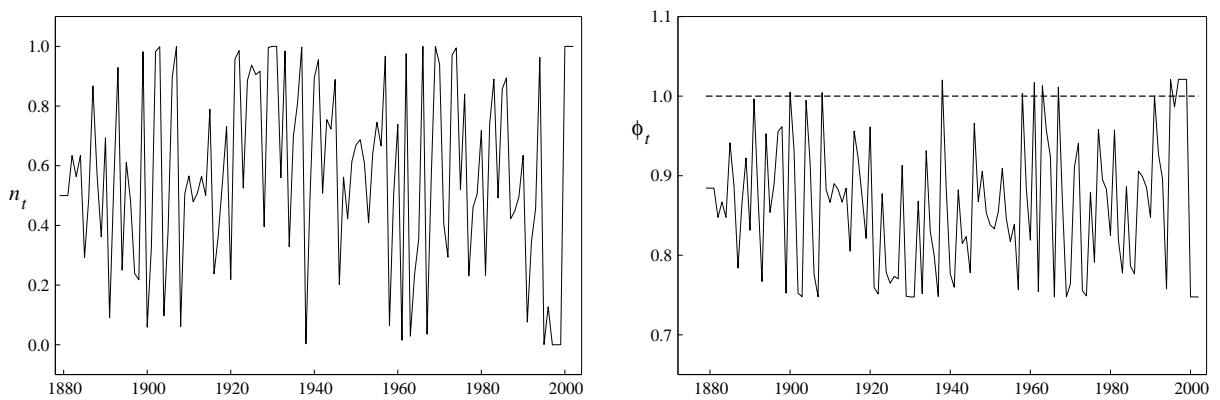

Figure 1.10. Estimated fraction $n_{t}$ of fundamentalists (left) and average extrapolation factor $\varphi_{t}$ (right) in (1.4.25) in 2-type model.

to grow over time at a constant speed larger than $R^{*} \approx 1.074$. At times when the fraction of investors using this belief is equal or close to 1 we have explosive behavior in the $\mathrm{P} / \mathrm{E}$ ratio. The sentiment of investors switches between a stable fundamentalists regime and a trend following regime. In normal periods agents consider the deviation as a temporary phenomenon and expect it to revert back to fundamentals quickly. In other periods, a rapid increase of stock prices not paralleled by improvements in the fundamentals causes losses for fundamentalists and profits for trend followers. Evolutionary pressure will then cause more fundamentalists to become trend followers, thus reenforcing the trend in prices.

Figure 1.10 shows the time series of the fraction of fundamentalists and the average market sentiment, defined as

$$
\varphi_{t}=\frac{n_{t} \varphi_{1}+\left(1-n_{t}\right) \varphi_{2}}{R^{*}}
$$

It is clear that the fraction of fundamentalists varies considerably over time with periods in which it is close to 0.5 and other periods in which it is close to either of the extremes 0 or 1 . The series of the average market sentiment shows that there is significant time variation between periods of strong mean reversion when the market is dominated by fundamentalist and other periods in which $\varphi_{t}$ is close to or exceeds 1 and the market is dominated by trend followers. These plots also offer an explanation of the events of the late 1990s: for six consecutive years the trend following strategy outperformed the fundamentalists strategy and a majority of agents switched to the trend following strategy, driving the average market sentiment beyond 1 thus reenforcing the strong price trend. However, at the turn of the market in 2000 the fraction of fundamentalists increased again, approaching 1 thus contributing to the reversal toward the fundamental value in subsequent years.

The estimation results show that there are two different belief strategies: one 
in which agents expect continuation of returns and the other in which they expect reversal. We also find that there are some years in which one type of expectations dominates the market. It is clear that the expectation of continuation of positive returns dominated the market in the late 1990s, with the average market sentiment coefficient $\varphi_{t}$ in (1.4.25)) larger than 1 in the late 1990s. Despite the awareness of the mispricing, in this period investors were aggressively extrapolating the continuation of the extraordinary performances realized in the past years. Our approach endogenizes the switching of agents among beliefs. The evolutionary mechanism that relates predictor choice to their past performance is supported by the data. It confirms also previous evidence that pointed in this direction. Based on answers to a survey, Shiller (2000) constructed indices of "Bubble Expectations" and of "Investor Confidence". In both cases, he finds that the time variation in the indices is well explained by the lagged change in stock prices. Based on a different survey, Fisher and Statman (2002) find that in the late 1990s individual investors had expectations of continuation of recent stock returns while institutional investors were expecting reversals. This is an interesting approach to identify heterogeneity of beliefs based on the type of investors rather than the type of beliefs. In the view of our model, the bubble in the 1990s was triggered by good news about economic fundamentals (a new internet technology), and strongly reinforced by trend extrapolating behaviour. The bubble reversed by bad news about economic fundamentals (excessive growth can not last forever and is not supported by earnings), and the crash was accelerated by switching of beliefs back to fundamentals.

\subsubsection{Empirical implications}

In this subsection we discuss some empirical implications of the estimation of our nonlinear evolutionary switching model with heterogeneous beliefs. First, we investigate the response to a positive shock to fundamentals when the asset is overvalued. Secondly, we address the question concerning the probability that a bubble may resume by considering the evolution of the valuation ratios conditional on data until the end of 2003. These simulation experiments both show the importance of considering nonlinear effects in the dynamics of stock prices.

\section{Response to a Fundamental Shock}

We use the estimated parameters to investigate the response of the market valuation to good news. Assume that at the beginning of period $t$ the cash flow increases due to a permanent increase in its growth rate. This implies that the asset has a higher fundamental valuation ratio, but what is the effect on the market valuation? We address this question both for the nonlinear switching model and a linear benchmark. The linear model may be interpreted as a model with a representative agent believing in an average mean reversion towards the fundamental ${ }^{26}$. Assume

\footnotetext{
${ }^{26}$ The linear benchmark is e.g. obtained for $\beta=0$ or equivalently, when both fractions $n_{t}=$ $1-n_{t}=0.5$ in (1.4.20). Hence, in the linear model there is no strategy switching between different
} 


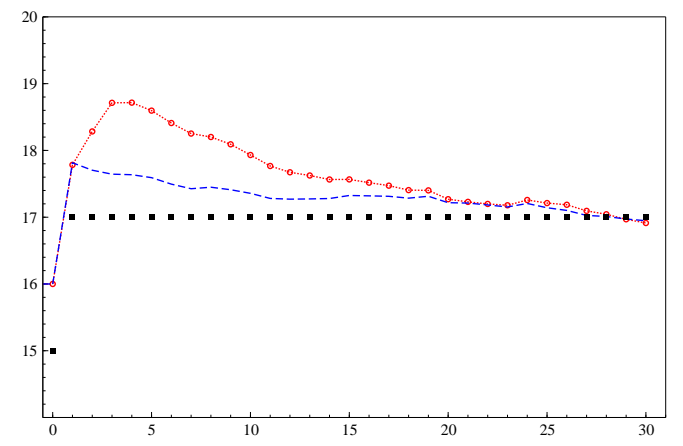

Figure 1.11. Average response (over 2000 simulations) to a shock to the fundamental for the linear representative agent model (dashed line) and the nonlinear 2-type switching model (dotted line with circles). At period 0 there is a permanent shock to the fundamental price from 15 to 17 . The simulation uses the estimated parameter values for the $\mathrm{P} / \mathrm{D}$-ratio, with a representative agent average belief parameter $\varphi=0.968$ and heterogeneous agent parameters $\varphi_{1}=0.762$ and $\varphi_{2}=1.135$ for the 2 types. The nonlinear heterogeneous agent model exhibits short-run continuation of positive returns and long-term reversal.

that at $t-1$ the fundamental valuation ratio was 15 and the good news at time $t$ drives it to 17 . Assume also that the equilibrium price at $t-1$ was 16 . Figure 1.11 shows the valuation ratio dynamics in response to the good news for both the linear and the nonlinear switching models.

The Figure shows the average price path over 2000 simulations of the estimated model. There is a clear difference between the linear and the nonlinear model. In the linear case, the positive shock to the fundamental value leads to an immediate increase of the price followed by mean-reversion thereafter. In contrast, for the nonlinear heterogeneous agent model, the pattern that emerges is consistent with the evidence of short-run continuation of positive returns and long-term reversal. After good news, the agents incorporate the news into their expectations and they expect that part of the previous period overvaluation will persist. One group -the trend followers- overreacts and expects a further increase of the price, while the other group -the fundamentalists- expects the price to diminish over time. The equilibrium price at time $t$ overshoots and almost reaches 18. In the following two periods trend followers continue to buy the stock and drive its price and its valuation ratio even higher. Finally, the reversal starts and drives the ratio back to its long run fundamental value. Initially, the aggressive investors interpret the positive news as a confirmation that the stock overvaluation was justified by forthcoming news.

types, but rather a representative agent with a linear forecasting rule. 

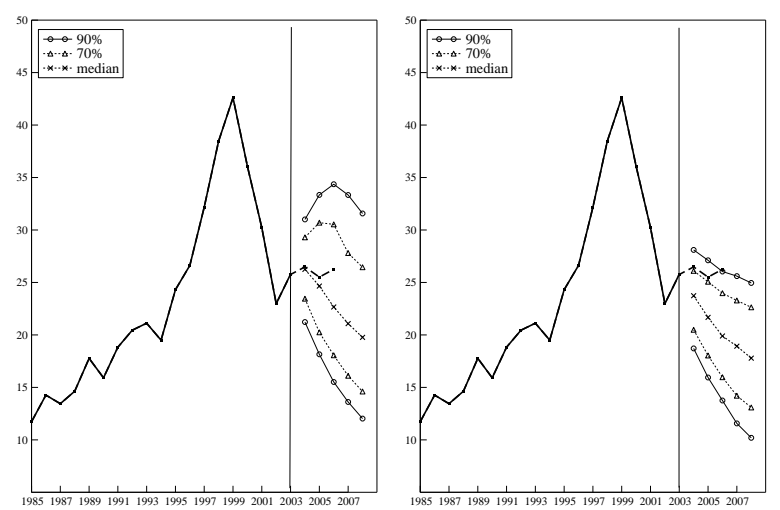

Figure 1.12. Prediction of $\mathrm{P} / \mathrm{E}$-ratios 5 years ahead, based on linear representative agent model (right) and nonlinear 2-type switching model (left). The estimated belief parameters are: $\varphi=0.983$ for the representative agent and $\varphi_{1}=0.80$ and $\varphi_{2}=1.097$ for the 2-type switching model. The quantiles corresponding to $10,30,50,70$ and $90 \%$ probability over 2000 replications are shown. The realized $\mathrm{P} / \mathrm{E}$-ratios 2004-2006 are also indicated, and show that the realized $\mathrm{P} /$ E-ratio for 2006 falls outside the $10 \%$ quantile of the linear model.

However, the lack of further good news convinces most investors to switch back to the mean reverting expectations and the stock price is driven back towards the fundamental.

\section{Will the bubble resume?}

As a model forecasting exercise, we simulate the evolution of the valuation ratios using the estimated heterogeneous agent model. We will then obtain the predicted evolution of the valuation ratio conditional on the value realized at the end of 2003. Innovations are obtained by reshuffling the estimated residuals. Instead of focusing our attention only on the mean or the median of the distribution we consider the quantiles corresponding to $10,30,50,70$ and $90 \%$ probability over 2000 replications of the estimated model in (1.4.24) for the $\mathrm{P} / \mathrm{E}$ ratio. In addition to the quantiles predicted by our nonlinear model we also plot those predicted by the linear representative agent model. Figure 1.12 shows the 1 to 5 periods ahead quantiles of the predictive distribution for the estimated model.

The linear model (right plot) predicts that the valuation ratio reverts back toward the mean at all quantiles considered. In contrast, the nonlinear switching model predicts that there is a significant probability that the ratio may increase again as a result of the activation of the trend following regime. The $70 \%$ and $90 \%$ quantiles clearly show that the PD-ratio may increase again to levels close to 
35. Stated differently, our heterogeneous agent model predicts that with probability over $30 \%$ the PD-ratio may increase to more than 30 . Note however that the median predicts that the ratio should decrease as implied by the linear mean reverting model. Another implication of our model is that if the first (mean reverting) regime dominates the beliefs of investors, it will enforce a much faster adjustment than predicted by the linear model. This is clear from the bottom quantiles of the distributions. These simulations show that predictions from a linear, representative agent model versus a nonlinear, heterogeneous agent model are quite different. In particular, extreme events with large deviations from the benchmark fundamental valuation are much more likely in a nonlinear world.

\subsection{LABORATORY EXPERIMENTS}

Asset pricing models with heterogeneous beliefs exhibit interesting dynamics characterized by temporary bubbles and crashes, triggered by news about fundamentals and reinforced by self-fulfilling expectations and trend following investment strategies. While the previous section focussed on the empirical relevance of such models, this section confronts the model with data from laboratory experiments with human subjects. Laboratory experiments are well suited to discipline the class of behavioral modes (or heuristics) boundedly rational subjects may use in economic decision making. Here, we discuss a number of "learning to forecast experiments", where subjects must forecast the price of an asset whose realized market price is an aggregation of individual expectations.

In real markets, it is hard to obtain detailed information about investors' individual expectations. One approach is to collect survey data on individual expectations, as done for example by Turnovsky (1970) on expectations about the Consumers' Price Index and the unemployment rate during the post-Korean war period. Frankel and Froot (1987ab, 1990ab), Allen and Taylor (1990), Ito (1990) and Taylor and Allen (1992) use a survey on exchange rate expectations, and conclude that financial practitioners use different forecasting and trading strategies. A consistent finding from survey data is that at short horizons investors tend to use extrapolative chartists' trading rules, whereas at longer horizons investors tend to use mean reverting fundamentalists' trading rules. Shiller $(1987,1990,2000)$ analyzes surveys on expectations about stock market prices and real estate prices and finds evidence for time variation in investors' sentiment; see also Vissing-Jorgensen (2003).

Laboratory experiments with human subjects provide an alternative, complementary approach to study the interaction of individual expectations and the resulting aggregate outcomes. An important advantage of the experimental approach is that the experimenter has full control over the underlying economic fundamentals. Surprisingly little experimental work focussing on expectation formation in markets has been done. Williams (1987) considers expectation formation in an experimental double auction market which varies from period to period by small shifts 
in the market clearing price. Participants predict the mean contract price for 4 or 5 consecutive periods. The participant with the lowest forecast error earns $\$ 1.00$. In Smith, Suchanek and Williams (1988) expectations and the occurrence of speculative bubbles are studied in an experimental asset market. In a series of papers, Marimon, Spear and Sunder (1993) and Marimon and Sunder (1993, 1994, 1995) studied expectation formation in inflationary overlapping generations economies. Marimon, Spear and Sunder (1993) find experimental evidence for expectationally driven cycles and coordination of beliefs on a sunspot 2-cycle equilibrium, but only after agents have been exposed to exogenous shocks of a similar kind. Marimon and Sunder (1995) present experimental evidence that a "simple" rule, such as a constant growth of the money supply, can help coordinate agents' beliefs and help stabilize the economy. Duffy $(2006,2007)$ gives stimulating surveys of laboratory experiments in various macro settings and how individual and aggregate behavior could be explained by agent-based models.

Most of these papers however can not be viewed as pure experimental testing of the expectations hypothesis, everything else being constant, because in the experiments dynamic market equilibrium is affected not only by expectations feedback but also by other types of human decisions, such as trading behavior. A number of laboratory experiments have focussed on expectation formation exclusively. Schmalensee (1976) presented subjects with historical data on wheat prices and asked them to predict the mean wheat price for the next 5 periods. In Dwyer et al. (1993) and Hey (1994) subjects had to predict a time series generated by a stochastic process such as a random walk or a simple linear first order autoregressive process; in the last two papers no economic context was given. Kelley and Friedman (2002) consider learning in an Orange Juice Futures price forecasting experiment, where prices are driven by a linear stochastic process with two exogenous variables (weather and competing supply). A drawback common to these papers is that the historical or stochastic price series are exogenous and there is no feedback from subjects' forecasting behavior.

\subsubsection{Learning to forecast experiments}

In the remaining part of this section we mainly focus on the learning to forecast experiments in Hommes et al. (2005). In these experiments, subjects forecast the price of a risky asset which is determined by market clearing with feedback from individual expectations. Similar experiments have been performed by van de Velden (2001), Gerber et al. (2002), Sutan and Willinger (2005), Adam (2007), Hommes et al. (2007) and Heemeijer (2007); see also the recent survey in Duffy (2007). We are particularly interested in the following questions:

1. How do boundedly rational agents form individual expectations and how do they learn in a heterogeneous world?

2. How do individual forecasting rules interact and what is the aggregate out- 
come of these interactions?

3. Will coordination occur, even when there is limited market information?

4. Does learning enforce convergence to rational expectations equilibrium?

In real financial markets traders are involved in two related activities: prediction and trade. Traders make a prediction concerning the future price of an asset, and given this prediction, they make a trading decision. In the experiments discussed here subjects' only task is to forecast prices; asset trading is computerized and derived from optimal demand (from mean-variance maximization), given the individual forecast. The experiments can therefore be seen as learning to forecast experiments (Marimon and Sunder 1994, p.134), in contrast to learning to solve intertemporal optimization problems or, more concisely, learning to optimize experiments (Duffy 2006, p.4), where participants are asked to submit their decisions (e.g. trading or consumption quantities), while their private beliefs about future developments remain implicit. Learning to forecast experiments provide us with 'clean' data on expectations, which can be used to test various expectations hypotheses.

In the experiments each participant is told that he is an advisor to a pension fund, with the only task to predict next period's price of a risky asset. Earnings are given by a (truncated) quadratic scoring rule

$$
e_{h t}=\max \left\{1300-\frac{1300}{49}\left(p_{t}-p_{h t}^{e}\right)^{2}, 0\right\} \text {, }
$$

where 1300 points is equivalent to 0.5 Euro, and earnings are zero in period $t$ when $\left|p_{t}-p_{h t}^{e}\right| \geq 7$. Subjects are informed that their pension fund needs to decide how much to invest in a risk free asset paying a risk free gross rate of return $R=1+r$, where $r$ is the real interest rate, and how much to invest in shares of an infinitely lived risky asset. The risky asset pays uncertain IID dividends $y_{t}$ with mean $\bar{y}$. The mean dividend $\bar{y}$ and the interest rate $r$ are common knowledge, so that the subjects could compute the (constant) fundamental $p^{*}=\bar{y} / r=3 / 0.05=60$. Subjects know that the price of the asset is determined by market clearing. Although they do not know the exact underlying market clearing equation, they have qualitative information about the market and are informed that the higher their forecast, the larger will be the fraction of money of their pension fund invested in the risky asset and the larger will be the demand for stocks. They do not know the exact investment strategy of their pension fund and the investment strategies of the other pension funds. They also do not know the number of pension funds (which is 6 ) or the identity of the other members of the group.

The experiment lasts for 51 periods. In every period $t$ the participants have to predict the price $p_{t+1}$ of the risky asset in period $t+1$, given the available information consisting of past prices $p_{t-1}, p_{t-2}, \ldots, p_{1}$ and the participants' own past individual predictions $p_{h t}^{e}, p_{h, t-1}^{e}, \ldots, p_{h 1}^{e}$. Notice that the participants have to make a two period ahead forecast for $p_{t+1}$, since $p_{t-1}$ is the latest price observation 
available. Subjects are told that their price forecast has to be between 0 and 100 for every period. In periods 1 and 2 no information about past prices is available. At the end of period $t$, when all predictions for period $t+1$ have been submitted, the participants are informed about the price in period $t$ and earnings for that period are revealed. On their computer screen the subjects are informed about their earnings in the previous period, total earnings, a table of the last twenty prices and their corresponding predictions and time series of the prices and their predictions. Subjects have no information about earnings and predictions of others.

\subsubsection{The price generating mechanism}

The asset market is populated by 6 pension funds and a small fraction of fundamentalist robot traders. Each pension fund $h$ is matched with a participant and makes an investment decision at time $t$ based upon this participant's prediction $p_{h, t+1}^{e}$ of the asset price. The fundamentalist trader always predicts the fundamental price $p^{f}$ and trades based upon this prediction.

The realized asset price in the experiment is determined by market clearing, with the pension fund's asset demand derived from mean-variance maximization given their advisor's forecast, as in the standard asset pricing model with heterogeneous beliefs (e.g. Campbell, Lo and MacKinlay (1997), Brock and Hommes (1998), see Subsection 1.1). The market clearing price is given by (cf. 1.1.5):

$$
p_{t}=\frac{1}{1+r}\left[\left(1-n_{t}\right) \bar{p}_{t}\right]
$$

where $\bar{p}_{t+1}^{e}=\frac{1}{6} \sum_{h=1}^{6} p_{h, t+1}^{e}$ is the average forecast for period $t+1$ of the 6 participants, $n_{t}$ is the time varying weight of the fundamentalist traders and $\varepsilon_{t}$ is a noise term, representing (small) stochastic demand and supply shocks. Note that the realized asset price $p_{t}$ at time $t$ is determined by the individual price predictions $p_{h, t+1}$ for time $t+1$. Therefore, when traders have to make a prediction for the price in period $t+1$ they do not know the price in period $t$ yet, and they can only use information on prices up till time $t-1$.

The weight $n_{t}$ of the fundamental traders in the market is endogenous and depends positively upon the absolute distance between the asset price and the fundamental value according to

$$
n_{t}=1-\exp \left(-\frac{1}{200}\left|p_{t-1}-p^{f}\right|\right) .
$$

The greater this distance the more the fundamental trader will buy or short the asset. The fundamentalist trader therefore acts as a 'stabilizing force' pushing prices in the direction of the fundamental price. Their presence excludes the possibility of ever lasting speculative bubbles in asset prices ${ }^{27}$. Also note that $n_{t}=0$, if $p_{t-1}=p^{f}$.

\footnotetext{
${ }^{27}$ DeGrauwe et al. (1993) discuss a similar stabilizing force in an exchange rate model with
} 

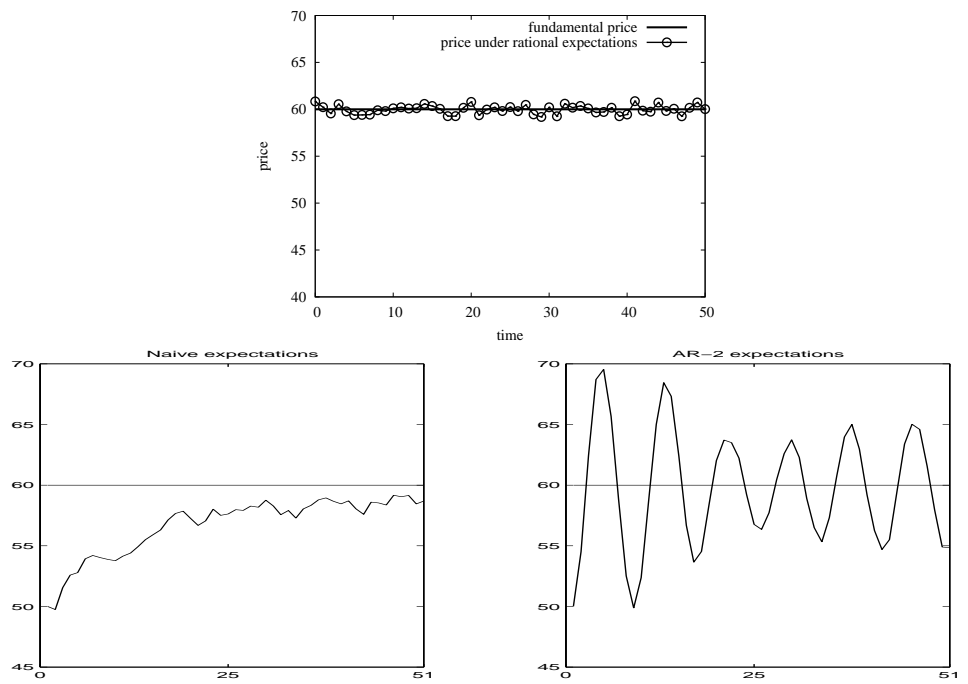

Figure 1.13. Realized prices under some benchmark expectation rules. Top panel: under rational expectations prices remain very close to the fundamental price 60 ; Bottom left: under naive expectations prices converge monotonically, but slowly, towards the fundamental price; Bottom right: under a simple linear AR-2 rule $p_{t+1}^{e}=\left(p_{t-1}+60\right) / 2+\left(p_{t-1}-p_{t-2}\right)$, which may be interpreted as an anchor and adjustment rule, prices exhibit persistent oscillations.

An important feature of the asset pricing model is its self-confirming nature or positive feedback: if all traders make a high (low) prediction the realized price will also be high (low). This feature is characteristic for speculative asset markets: if traders expect a high price, the demand for the risky asset will be high, and as a consequence the realized market price will be high, assuming that the supply is fixed.

\subsubsection{Benchmark expectations rules}

Figure 1.13 shows the price dynamics under three benchmark expectation rules: rational expectations, naive expectations and a trend extrapolation rule. In the rational expectations benchmarks all agents forecast the price to be equal to its

fundamentalists and chartists. In the same spirit Kyle and Xiong (2001) introduce a long-term investor that holds a risky asset in an amount proportional to the spread between the asset price and its fundamental value. Since in the experiments the fundamental value is $p^{f}=60$, the weight of the fundamentalist traders is bounded above by $\bar{n}=1-\exp \left(-\frac{3}{10}\right) \approx 0.26$. The weight of the other traders is the same for each trader and equal to $\left(1-n_{t}\right) / 6 \leq 0.17$. 
fundamental value $p^{f}=60 .{ }^{28}$ Realized prices are then given by

$$
p_{t}=p^{f}+\frac{1}{1+r} \varepsilon_{t}
$$

Hence, under rational expectations prices exhibit small random fluctuations around the fundamental price $p^{f}=60$. This outcome of the experiment should probably not be expected right from the start, but perhaps subjects can learn to coordinate on the rational, fundamental forecast.

Under naive expectations all participants use the last observed price as their forecast, that is, $p_{h, t+1}^{e}=p_{t-1}$. The asset price then converges monotonically towards the fundamental price, as illustrated in Figure 1.13. Finally, Figure 1.13 also illustrates what happens when all subjects use the simple trend extrapolation rule

$$
p_{h, t+1}^{e}=\frac{\left(60+p_{t-1}\right)}{2}+p_{t-1}-p_{t-2} .
$$

If all subjects use the forecasting rule (1.5.5), realized market prices will fluctuate for 50 periods. This simple rule may be viewed as an anchor and adjustment heuristic, following the terminology of Tversky and Kahnemann (1974), since it uses an anchor (the average of the fundamental price and the last observed price) and extrapolates the last price change from there. One may wonder how subjects would arrive at this anchor, if they do not know the fundamental, but quite surprisingly a number of subjects used a rule very similar to (1.5.5).

\subsubsection{Aggregate behavior}

Figure 1.14 shows time series of the realized asset prices and individual predictions in the experiments for five different groups. The first three groups illustrate the three typical qualitatively different outcomes in the treatment with robot traders:

i) monotonic convergence: the price converges monotonically to the fundamental price from below;

ii) persistent oscillations: the price oscillates with more or less constant amplitude; there is no convergence of the price to its fundamental value.

iii) dampened oscillations: the price oscillates around the fundamental price, with large amplitude initially but the amplitude decreases over time indicating (slow) convergence to the fundamental price.

The last two groups in Figure 1.14 illustrate what happens in a different treatment of the experiments without fundamental robot traders. When there are no fundamental robot traders present in the market, persistent price oscillations with large amplitude typically occur. The difference between these last two groups lies in

\footnotetext{
${ }^{28}$ Recall that participants know the values of $\bar{y}$ and $r$, and therefore have enough information to compute the fundamental value and predict it for any period.
} 

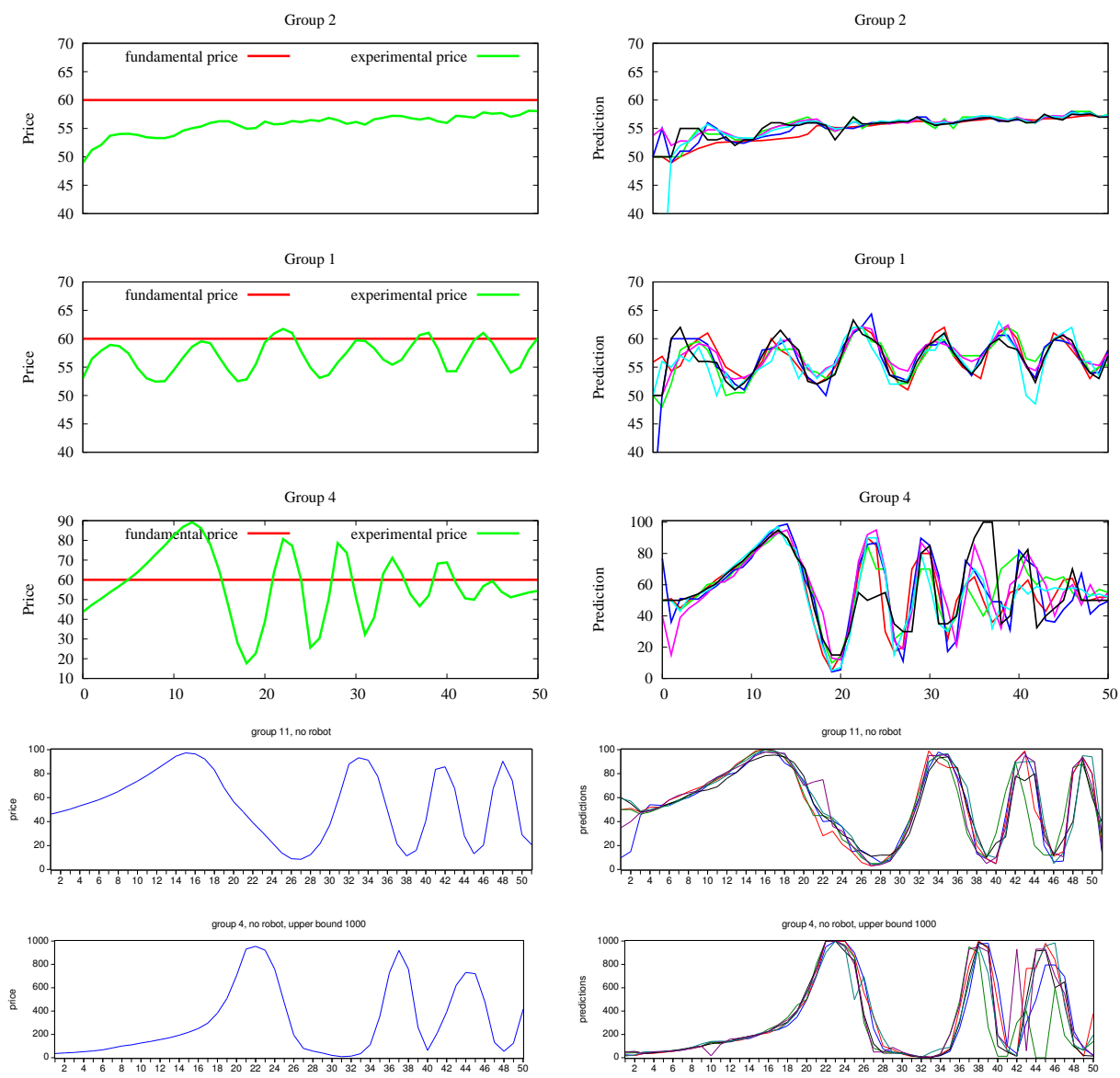

Figure 1.14. Realized prices (left) and individual predictions (right) in five typical asset pricing experiments. The fundamental price $p^{f}=60$ is indicated by a horizontal line. The first three examples illustrate three different outcomes in the experiments with robot traders: monotonic convergence, persistent oscillations and dampened oscillations. The last two examples show experiments without robot traders for upper bounds of 100 and 1000 respectively. 
the upper bound for price predictions, set to 100 (as in the case with robot traders) and 1000 respectively. Hommes et al. (2008) ran experiments without robot traders and a high upper bound of 1000 (maintaining the same fundamental price $p^{f}=60$ ) and in 6 out of their 7 markets long lasting price bubbles (almost) reaching the upper bound were observed, with price levels up to 15 times the fundamental value.

Comparing the experimental results in Figure 1.14 with the simulated benchmarks in Figure 1.13 one observes that realized prices under naive expectations resemble realized prices in the case with monotonic convergence remarkably well. On the other hand, the case of persistent oscillatory behavior in the experiment is qualitatively similar to the asset price behavior when participants use a simple $A R(2)$ prediction strategy. Clearly, naive and $A R(2)$ prediction strategies give a qualitatively much better description of aggregate asset price fluctuations in the experiment than does the benchmark case of rational expectations. Recall from Subsection 1.5.3 that an $A R(2)$ rule has a simple behavioral interpretation as an anchor and adjustment trend following forecasting strategy.

\subsubsection{Individual prediction strategies}

In this subsection we discuss some characteristics and estimation of individual prediction strategies. Some participants try to extrapolate observed trends and by doing so overreact and predict too high or too low. Other participants are more cautious when submitting predictions, using adaptive expectations, that is, an average of their last forecast and the last observed price. An individual degree of overreaction can be quantified as the average absolute (one-period) change in predictions of participant $h$ :

$$
\triangle_{h}^{e}=\frac{1}{41} \sum_{t=11}^{51}\left|p_{h t}^{e}-p_{h, t-1}^{e}\right|
$$

The average absolute change in the price is given by $\triangle=\frac{1}{41} \sum_{t=11}^{51}\left|p_{t}-p_{t-1}\right|$. We will say that individual $h$ overreacts if $e=\triangle$ and we will say that individual $h$ is cautious if $\triangle_{h}^{e} \leq \triangle$. Figure 1.15 illustrates the individual degree of overreaction for the different groups. In the case of monotonic convergence (groups 2 and 5), there is no overreaction; in the case of permanent oscillations (groups 1, 6, 8, 9) a majority of subjects shows some overreaction, but it is relatively small. In the case of dampened oscillations (groups 4, 7 and 10), with large temporary bubbles in the initial phases of the experiment, a majority of participants strongly overreacts. Oscillatory behavior and temporary bubbles are thus caused by overreaction of a majority of agents.

Individual prediction strategies have been estimated using a simple linear model

$$
p_{h, t+1}^{e}=\alpha_{h}+\sum_{i=1}^{4} \beta_{h i} p_{t-i}+\sum_{j=0}^{3} \gamma_{h j} p_{h t-j}^{e}+\nu_{t},
$$




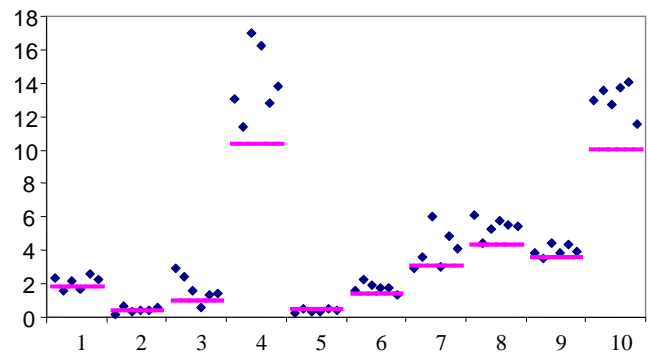

Figure 1.15. Individual degrees of overreactions for 10 different groups, all with a robot trader, the first 7 with a fundamental $p^{f}=60$ and the last 3 with a fundamental $p^{f}=40$. The line segments represent the average absolute price change, while the dots represent the average absolute changes in individual forecasts. Dots above the line segments correspond to individual overreaction.

where $\nu_{t}$ is an IID noise term. This general setup includes several important special cases: $i)$ naive expectations ( $\beta_{h 1}=1$, all other coefficients equal to 0$)$; ii) adaptive expectations $\left(\beta_{h 1}+\gamma_{h 0}=1\right.$, all other coefficients equal to 0$)$ and iii) $A R(L)$ processes (all coefficients equal to 0 , except $\alpha_{h}, \beta_{h 1}, \ldots, \beta_{h L}$ ). The estimation results for 60 participants (using observations $t=11$ to $t=51$ ) may be summarized as follows:

1. For more than $90 \%$ of the individuals the simple linear rule (1.5.7) describes forecasting behavior well;

2. In the monotonically converging markets, a majority of subjects uses a naive, an adaptive or an $\mathrm{AR}(1)$ forecasting rule;

3. In the dampened and persistently oscillating markets a majority of subjects uses simple $\mathrm{AR}(2)$ or $\mathrm{AR}(3)$ forecasting rules; in particular a number of subjects use a simple trend following rule of the form

$$
p_{h, t+1}^{e}=p_{t-1}+\delta_{h}\left(p_{t-1}-p_{t-2}\right), \quad \delta_{h}>0 .
$$

This forecasting rule corresponds to positive feedback or momentum traders.

Within each group, participants learn to coordinate on a simple forecasting rule, which becomes self-fulfilling. If participants coordinate on an adaptive or $\operatorname{AR}(1)$ forecasting rule, the asset price monotonically converges to the fundamental price. In contrast, if the participants coordinate on a trend following rule, transitory or even permanent price oscillations may arise, with persistent deviations from fundamental price. Anufriev and Hommes (2007) extended the adaptive belief systems (ABS) in Section 1.1 and developed an evolutionary heuristics switching 
model matching all three different observed patterns in the learning to forecasting experiments remarkably well.

\subsubsection{Profitability}

In the learning to forecasting experiments subjects have been rewarded by their forecasting performance. As discussed in Hommes (2001), the fitness measure of (minus) squared forecasting errors is equivalent to risk adjusted profits and therefore it may be a relevant measure in real markets (cf. footnote 6). But it is interesting to investigate the corresponding realized profits of the pension funds. In this section we therefore briefly discuss the profitability, that is the (non-risk adjusted) realized profits, of the investment strategies. Realized profits of a mean-variance investment strategy based on a price forecast $p_{h, t+1}^{e}$ are given by

$$
\pi_{h t}=\left(p_{t+1}+y_{t+1}-R p_{t}\right)\left(p_{h, t+1}^{e}+\bar{y}-R p_{t}\right)
$$

As a typical example, Figure 1.16 shows the realized profits and the realized accumulated profits in group 4, that is, a group with robot trader and upperbound 100 exhibiting dampened price oscillations (the third panel in Figure 1.14). Figure 1.16 shows the realized profits corresponding to the six individual forecasts, the realized profits of the fundamental robot trader, the realized profits of a hypothetical switching strategy, together with the realized price series (scaled by a factor 3 ).

Clearly there are large fluctuations in the realized profits and all strategies occasionally suffer from large losses. The fundamental strategy starts with positive profits in periods $1-6$, as the asset price rises from below the fundamental. When the asset price rises above its fundamental value and the bubble starts, the fundamental strategy makes large losses in periods $7-13$. At the peak of the first bubble, at period 13, the fundamental strategy has performed one but last on average, with the one but lowest accumulated realized profits. During the crash however, the fundamentalists make huge profits because they have built a large short position in the risky asset. At the same time, most other (trend following) strategies suffer large losses, because they hold long positions. As the crash continues and the asset price falls below its fundamental value, the fundamental strategy starts making losses again. At the bottom of the market in period 19, fundamentalists make a large loss. Over the full sample of 50 periods however, on average the fundamental strategy performs very well and accumulates more profits than the other 6 forecasting strategies. Figure 1.16 also shows that the fundamental strategy is beaten by a switching strategy, always selecting the best (according to last period's realized profit) out of the 7 other strategies. Stated differently, the switching strategy always uses the forecast of the advisor whose pension fund generated highest realized profit in the previous period. Such a switching strategy beats the fundamental robot strategy. 

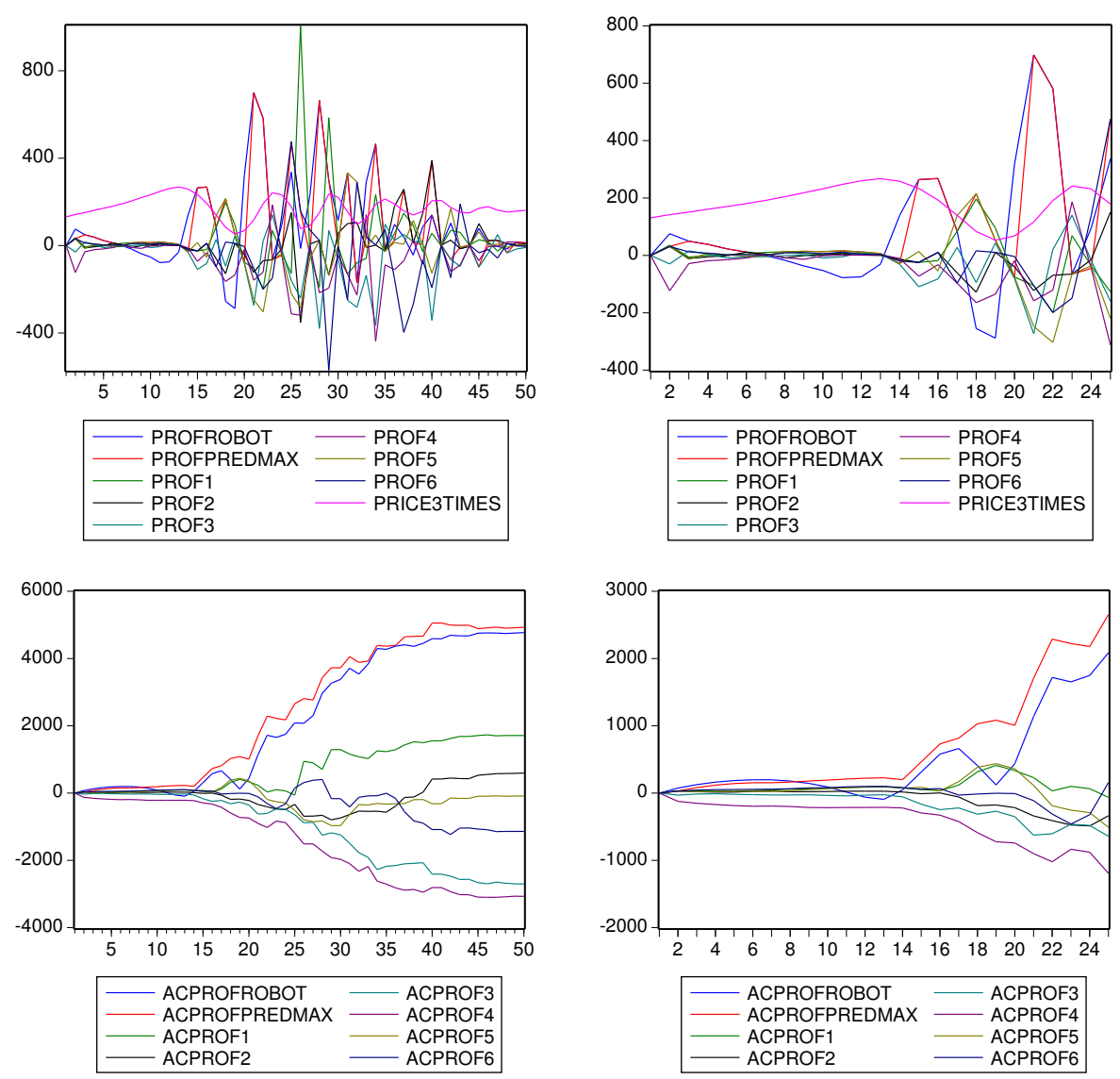

Figure 1.16. Realized (non-risk adjusted) profits (top panel) and accumulated profits (bottom panel) for group 4 (see Fig. 1.14), with a robot trader and price upper bound 100. The left panels show periods 1-50, while right panels zoom into periods 1-25; the realized price series (scaled by a factor 3 ) is also shown. The top panel shows graphs of the realized profits corresponding to the six individual forecasting strategies, the fundamental robot trader, and a hypothetical switching strategy using the best (according to last period's realized profits) of the other 7 strategies. The bottom panel shows the accumulated profits of these 8 strategies. Realized profits exhibit large fluctuations over time and all strategies at times suffer from large losses. On average and in terms of accumulated profits the fundamental strategy performs very well, but is beaten by the switching strategy. 


\subsection{CONCLUDING REMARKS}

This chapter has reviewed some behavioral finance models with evolutionary selection of heterogeneous trading strategies and discussed their empirical and experimental validity. When strategy selection is driven by short run realized profits, trend following strategies may destabilize asset markets. Asset price fluctuations are characterized by phases where fundamentalists dominate and prices are close to fundamentals, suddenly interrupted by possibly long lasting phases of price bubbles when trend following strategies dominate the market and prices deviate persistently from fundamentals. Even in simple heterogeneous beliefs models, asset prices are difficult to predict and market timing based on the prediction of the start or the collapse of a bubble is extremely difficult and highly sensitive to noise. Estimation of simple versions of heterogeneous agent models on yearly S\&P500 data suggests that stock prices are characterized by behavioral heterogeneity. Simple evolutionary models therefore could provide an explanation of, for example, the "dot com" bubble, as being triggered by good news about economic fundamentals, and subsequently strongly amplified by trend following trading strategies. Laboratory experiments with human subjects confirm that coordination on simple trend following strategies may arise in asset markets and cause persistent deviations from fundamentals.

In a heterogeneous beliefs asset pricing model, as long as prices fluctuate around their fundamental value, fundamentalists strategies do quite well in terms of accumulated profits. If there are no limits to arbitrage and fundamentalists can survive possibly long lasting bubbles during which they suffer large losses, their strategy performs very well in the long run and may help stabilize markets. However, fundamentalists can be beaten by a switching strategy based on recent realized profits, thus providing an incentive for investors to keep switching strategies. It should be noted that the models discussed here are very stylized with a well defined fundamental price. In real markets, there may be a lot of disagreement about the "correct" fundamental price and it may then be not so clear what the fundamental strategy would be. Limits to arbitrage may also prevent fundamentalists from holding long lasting positions opposite to the trend, as more and more traders go with the trend based on their recent success.

Most behavioral asset pricing models focus on a single risky asset. Only few extensions to a multi-asset setting have been made until now. Westerhoff (2004) and Chiarella et al. (2007) considered multi-asset markets, where chartists can switch their investments between different markets for risky assets. The interaction between the different markets causes complex asset price dynamics, with different markets exhibiting co-movements as well as clustered volatility and fat tails of asset returns. Böhm and Wenzelburger (2005) apply random dynamical systems to investigate the performance of efficient portfolios in a multi-asset market with heterogeneous investors.

Work on complex evolutionary systems in finance is rapidly growing, but little 
work has been done on policy implications so far. The most important difference with a representative rational agent framework is probably that in a heterogeneous boundedly rational world, asset price fluctuations exhibit excess volatility. If this is indeed the case, it has important policy implications e.g. concerning the debates on whether a Tobin tax on financial transactions is desirable or whether financial regulation is desirable. Westerhoff and Dieci (2006) use a complex evolutionary system to investigate the effectiveness of a Tobin tax. Investors can invest in two different speculative asset markets. If a Tobin tax is imposed on one market, it is stabilized while the other market is destabilized; if a tax is imposed on both markets, price fluctuations in both markets decrease. Brock, Hommes and Wagener (2008) study the effects of financial innovation upon price volatility and welfare. They extend the asset pricing model with heterogeneous beliefs in Section 1.1 by introducing hedging instruments in the form of Arrow securities, that is, state contingent claims to uncertain future events. They show that more hedging instruments may destabilize markets and decrease welfare when agents are boundedly rational and choose investment strategies based on reinforcement learning. The intuition of this result is simple: optimistic and/or pessimistic traders take larger positions when they can hedge more risk, and those who happen to be on the right side of the market will be reinforced more. In a world of bounded rationality and learning by past success, more hedging instruments may thus lead to more persistent deviations from market fundamentals. Developing a theory of complex multi-asset market models with heterogeneous interacting trading strategies and its empirical and experimental testing will be an important area of research for years to come. From a practitioners viewpoint this kind of research seems highly relevant to gain more insight into the causes of financial crises, such as the recent credit crisis, in order to hopefully avoid them in the future. 


\section{APPENDIX ON BIFURCATION THEORY}

The purpose of this appendix is to show how stability loss of a stable steady state is necessarily connected to one of a small number of standard bifurcations. For this, we sketch in the first section how a system at stability loss can be reduced to a one- or two-dimensional system. In the second section we present the most common bifurcation scenarios, saddle-node, period-doubling, Hopf and pitchfork. See Kuznetsov (1995) for a detailed mathematical treatment of bifurcation theory.

\section{A.1 BASIC CONCEPTS FROM DYNAMICAL SYSTEMS.}

Instead of directly dealing with evolution equations of the form

$$
x_{t}=\varphi\left(x_{t-1}, \cdots, x_{t-n}\right)
$$

dynamical system theory usually considers first order vector dynamics

$$
y_{t}=\Phi\left(y_{t-1}\right)
$$

where $y_{t}=\left(x_{t}, \cdots, x_{t-(n-1)}\right)$ and where the system map $\Phi: \mathbb{R}^{n} \rightarrow \mathbb{R}^{n}$ is given as

$$
\Phi\left(y_{t}\right)=\left(\begin{array}{c}
\varphi\left(x_{t-1}, \cdots, x_{t-n}\right) \\
x_{t-1} \\
\vdots \\
x_{t-(n-1)}
\end{array}\right) .
$$

Given an initial state $y_{0}$, the orbit of $\Phi$ through $y_{0}$ is the sequence $\left\{y_{t}\right\}_{t=0}^{\infty}$ satisfying A.1.2 for every $t$. For instance a steady state $\bar{x}$ of the evolution equation, which satisfies $\bar{x}=\varphi(\bar{x}, \cdots, \bar{x})$, corresponds to the constant orbit $y_{t}=(\bar{x}, \cdots, \bar{x})$ for all $t$ of $\Phi$. Such an orbit is called a fixed point of the dynamical system $\Phi$.

In this appendix we discuss only stability changes of fixed points (but stability changes of periodic points can be handled similarly). By definition, a fixed point $\bar{y}$ is asymptotically stable if for all $y_{0}$ sufficiently close to $\bar{y}$ the orbit of $\Phi$ through $y_{0}$ tends to $\bar{y}$ as $t \rightarrow \infty$. A fixed point $\bar{y}$ is unstable, if arbitrarily close to it there are initial points $y_{0}$ whose orbits do not tend to $\bar{y}$ as $t \rightarrow \infty$.

Let us assume, for simplicity, that $y=0$ is a fixed point of $\Phi$. The linearisation of the dynamics A.1.2 at $y=0$ is given as

$$
y_{t}=L y_{t-1}
$$

where $L=D \Phi(0)$ is the $n \times n$ Jacobi matrix of $\Phi$ at $\bar{y}=0$. We have

Theorem A.1.1 If all eigenvalues $\lambda_{j}, j=1, \cdots, n$, of $L$ satisfy $\left|\lambda_{j}\right|<1$, then the fixed point $y=0$ is asymptotically stable. If there is at least one eigenvalue such that $\left|\lambda_{j}\right|>1$, then $y=0$ is unstable. 
In general, a fixed point $\bar{y}$ is called hyperbolic if no eigenvalue $\lambda$ of $L$ is on the complex unit circle, that is, $|\lambda| \neq 1$. Note that the stability of a hyperbolic fixed point can be determined just by looking at the eigenvalues.

A bifurcation of a system is a qualitative change in the orbit structure as a system parameter is changed. In this appendix, we shall focus on the simplest kind of bifurcations, the possibilities of a fixed point to lose stability as a one-dimensional parameter is varied.

That is, we consider the case that the system map $\Phi_{\mu}: \mathbb{R}^{n} \rightarrow \mathbb{R}^{n}$ depends on a parameter $\mu \in\left[\mu_{1}, \mu_{2}\right]$. Let us assume that $\bar{y}_{1}$ is an attracting hyperbolic fixed point of the map $\Phi_{\mu_{1}}$. Note that the fixed point equation

$$
\Phi_{\mu}(y)-y=0
$$

can be solved for $y$ as a function of $\mu$ whenever $\operatorname{det}\left(D \Phi_{\mu}(y)-I\right) \neq 0$, that is, whenever $\lambda=1$ is not an eigenvalue of $D \Phi_{\mu}(y)$. Consequently, we can find a parametrised family of fixed points $\bar{y}_{\mu}$ such that $\bar{y}_{\mu_{1}}=\bar{y}_{1}$, and we can investigate the stability of $\bar{y}_{\mu}$ as $\mu$ varies.

The eigenvalues $\lambda_{1}, \cdots, \lambda_{n}$ of $L_{\mu}=D \Phi_{\mu}\left(\bar{y}_{\mu}\right)$ are continuous functions of $\mu$; by assumption $\left|\lambda_{j}\left(\mu_{1}\right)\right|<1$ for all $j$. Theorem A.1.1 implies that stability changes can only occur if for some $\mu_{0} \in\left(\mu_{1}, \mu_{2}\right)$ the fixed point fails to be hyperbolic; that is, if one of the eigenvalues, say $\lambda_{1}\left(\mu_{0}\right)$, has absolute value one.

There are three main mechanisms how a fixed point can fail to be hyperbolic: the saddle-node bifurcation $\lambda_{1}\left(\mu_{0}\right)=1$; the period-doubling bifurcation $\lambda_{1}\left(\mu_{0}\right)=-1$; and the Hopf bifurcation $\lambda_{1}\left(\mu_{0}\right)=\mathrm{e}^{\mathrm{i} \alpha}$ with $0<\alpha<\pi$. In the last case, there are two eigenvalues of absolute value equal to one, as the complex conjugate $\mathrm{e}^{-\mathrm{i} \alpha}$ is necessarily an eigenvalue as well. Of course, it could happen that several eigenvalues have absolute value equal to one simultaneously in configurations other than those listed above, but it can be shown that these cases are atypical for one-parameter systems. $^{29}$

We shall analyse these three bifurcations one by one below. The first step in each analysis is to simplify the system by restricting it to an invariant centre manifold. Recall that a set $W$ is invariant under $\Phi$ if $\Phi(W)=W$.

Theorem A.1.2 (Centre manifold theorem). Let $y_{\mu}$ be a fixed point of $\Phi_{\mu}$ that is nonhyperbolic if $\mu=\mu_{0}$. For $\mu$ sufficiently close to $\mu_{0}$, there is a family of invariant manifolds $W_{\mu}^{c}$, depending differentiably on $\mu$, such that $W_{\mu_{0}}^{c}$ is tangent to the eigenspace associated to the nonhyperbolic eigenvalues at $\mu=\mu_{0}$.

The complete complexity of a bifurcation is retained if the map $\Phi_{\mu}$ is resticted to the centre manifold $W_{\mu}^{c}$.

\footnotetext{
${ }^{29}$ These configurations are non-persistent: they can be removed by making an arbitrarily small change to the system $\Phi_{\mu}$.
} 


\section{A.2 BIFURCATION SCENARIOS}

\section{The saddle-node bifurcation}

At this bifurcation, two fixed points are created or disappear, depending on the direction of the parameter change.

A saddle node bifurcation occurs in the case that $\lambda_{1}\left(\mu_{0}\right)=1$ and a onedimensional associated eigenspace. After introducing suitable new variables, the restriction of $\Phi_{\mu}$ to the associated one-dimensional centre manifold takes the form

$$
\Phi_{\mu}(x)=x+\mu-x^{2}+x^{3} g(x, \mu),
$$

where $g$ is some differentiable function. In fact, it is sufficient to consider the normal form

$$
\Phi_{\mu}^{\mathrm{NF}}(x)=x+\mu-x^{2}
$$

this has to be justified afterwards by arguing that the full family $\Phi_{\mu}$ has qualitatively the same dynamics. This latter step is not hard but rather technical and will therefore be omitted.

Let us analyse the normal form. Note that for $\mu=0$, the system has a single fixed point $x=0$ and $L=D \Phi_{0}^{\mathrm{NF}}(0)=1$, so we are indeed in the saddle-node case. For general $\mu$, fixed points are the solutions of the equation $x=\Phi_{\mu}^{\mathrm{NF}}(x)$; for $\mu \geq 0$ they are given as

$$
\bar{x}_{1}=\sqrt{\mu}, \quad \bar{x}_{2}=-\sqrt{\mu},
$$

whereas for $\mu<0$ no fixed points exist.

The linearisations $L_{j}=D \Phi^{\mathrm{NF}}\left(\bar{x}_{j}\right)=1-2 \bar{x}_{j}$ read as

$$
L_{1}=1+2 \sqrt{\mu}, \quad L_{2}=1-2 \sqrt{\mu} .
$$

We conclude that $\bar{x}_{1}$ is repelling and that $\bar{x}_{2}$ is attracting.

All this information can be summarised in a bifurcation diagram; see figure A.17. In the diagram, the location of the fixed points is plotted as a function of the parameter $\mu$; the branch of stable fixed points is indicated by the solid curve, whereas the dashed curve indicates the location of unstable fixed points. At $\mu=0$, the two branches meet and there is exactly one fixed point; for $\mu<0$, there are no fixed points.

\section{The period doubling bifurcation}

In this bifurcation, an attracting fixed point loses stability, and a period-two orbit is generated.

A period doubling bifurcation occurs if $\lambda_{1}\left(\mu_{0}\right)=-1$. The eigenspace and the corresponding centre manifold are again one-dimensional. The normal form reads in this case as

$$
\Phi_{\mu}^{\mathrm{NF}}(x)=-x-\mu x+a x^{3}
$$




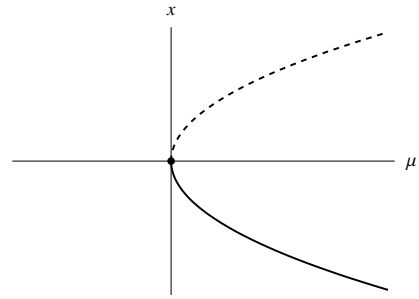

(a) Saddle-node

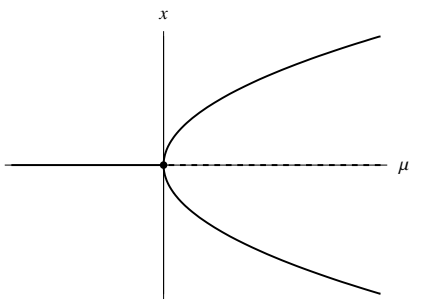

(b) Period-doubling/pitchfork

Figure A.17. Saddle-node and period-doubling/pitchfork bifurcation diagrams

with $a= \pm 1$. We only consider the supercritical case $a=1$, which occurs if the fixed point is globally attracting under $\Phi_{\mu}$ for $\mu<0$. Note that $\bar{x}=0$ is now a fixed point for all values of $\mu$; moreover,

$$
L=D \Phi_{\mu}^{\mathrm{NF}}(0)=-1-\mu .
$$

We conclude that $\bar{x}$ is attracting for $-2<\mu<0$ and repelling for $\mu>0$ or $\mu<-2$, implying in particular that the fixed point loses its stability at $\mu=0$. Moreover, note that

$$
\left|\Phi^{\mathrm{NF}}(|x|)\right|=\left(1+\mu-|x|^{2}\right)|x|=|x|
$$

if $|x|=\sqrt{\mu}$. This implies that the set $P=\{\sqrt{\mu},-\sqrt{\mu}\}$ is invariant. As the points in this sets are not fixed points, necessarily one is mapped to the other by the $\operatorname{map} \Phi^{\mathrm{NF}}$.

The set $P$ is a so-called periodic orbit, and its elements are period- 2 points. In general, a period- $m$ point is a point which is mapped to itself after $m$ iterations of the system map. It can be verified that nearby orbits are attracted to the period 2 orbit $P$ as $t \rightarrow \infty$; the period 2 orbit $P$ is asymptotically stable.

\section{The Hopf bifurcation}

In a Hopf (or Neimark-Sacker) bifurcation, a fixed point loses stability and an invariant circle is generated. The bifurcation occurs if $\lambda_{1}\left(\mu_{0}\right)=\mathrm{e}^{\mathrm{i} \alpha}$ and $\lambda_{2}\left(\mu_{0}\right)=$ $\mathrm{e}^{-\mathrm{i} \alpha}, \alpha \in(0, \pi) \backslash\left\{\frac{\pi}{3}, \frac{\pi}{2}, \frac{2 \pi}{3}\right\}$; the associated centre manifold is two-dimensional. Again restricting to the supercritical case, the normal form can be expressed as

$$
\Phi^{\mathrm{NF}}\left(x_{1}, x_{2}\right)=\left(1+\mu-x_{1}^{2}-x_{2}^{2}\right)\left(\begin{array}{rr}
\cos \vartheta(x) & -\sin \vartheta(x) \\
\sin \vartheta(x) & \cos \vartheta(x)
\end{array}\right)\left(\begin{array}{l}
x_{1} \\
x_{2}
\end{array}\right)
$$

where $\vartheta(x)=\alpha+\beta\left(x_{1}^{2}+x_{2}^{2}\right)$. By introducing polar coordinates $x_{1}=r \cos \psi$, $x_{2}=r \sin \psi$, the map takes the form

$$
\Phi^{\mathrm{NF}}(r, \psi)=\left(\begin{array}{c}
(1+\mu) r-r^{3} \\
\psi+\alpha+\beta r^{2}
\end{array}\right)
$$


As in the case of the period-doubling bifurcation, the origin $r=0$ is stable if $-2<$ $\mu<0$ and unstable if $\mu>0$ (or $\mu<-2$ ). Moreover, for $\mu>0$ there is a stable invariant circle

$$
C=\{(r, \psi): r=\sqrt{\mu}\} .
$$

That is, as the stable fixed point $r=0$ loses stability, a stable invariant circle branches off. The dynamics on the circle are given as

$$
\psi \mapsto \psi+\alpha+\beta \mu \bmod 2 \pi
$$

In the case of the Hopf bifurcation, investigating which properties of the normal form carry over to the full normal form is a nontrivial problem.

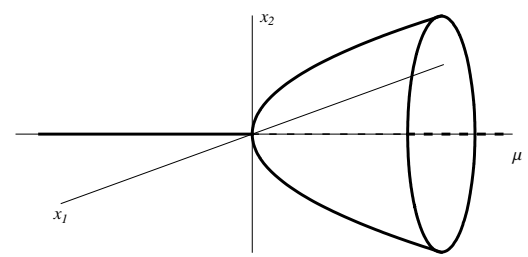

Figure A.18. Hopf bifurcation diagram

\section{The pitchfork bifurcation}

Above we have listed the three simplest typical bifurcations of general systems. Sometimes a system has a special symmetry, like a reflection symmetry: $\Phi(-x)=$ $-\Phi(x)$. In the space of such systems, bifurcations that are nontypical for general systems may become typical. An example is the pitchfork bifurcation, which is typical for systems with reflection symmetry.

The normal form for a (supercritical) pitchfork bifurcation reads as

$$
\Phi(x)=(1+\mu) x-x^{3} .
$$

Note that $\Phi(-x)=-\Phi(x)$, and that as a consequence the point $x=0$ is a fixed point for all $\mu$, stable if $-2<\mu<0$, unstable otherwise. Moreover, for $\mu>0$, the points $x= \pm \sqrt{\mu}$ are stable fixed points, branching off the fixed point $x=0$ as that point loses stability.

Note that the bifurcation diagram of the pitchfork bifurcation is identical to that of the period doubling bifurcation, but that the interpretation of the branches is different. In a (supercritical) pitchfork bifurcation one steady state changes stability, while two new (stable) steady states are created. 


\section{BIBLIOGRAPHY}

[1] Adam, K., (2007), Experimental evidence on the persistence of output and inflation, Economic Journal, 117, 603-636.

[2] Alchian, A.A., (1950), Uncertainty, evolution, and economic theory, Journal of Political Economy, 58, 211-221.

[3] Alfarno, S., Lux, T. and Wagner, F. (2005), Estimation of agent-based models: the case of an asymmetric herding model, Computational Economics 26, $19-49$.

[4] Allen, H. and Taylor, M.P., (1990), Charts, noise and fundamentals in the London foreign exchange market, Economic Journal 100, No. 400, Conference Papers, 49-59.

[5] Amir, R., Evstigneev, I.V., Hens, T. and Schenk-Hoppé, K.R. (2005), Market selection and survival of investment strategies, Journal of Mathematical Economics 41, 105-122.

[6] Anderson, P.W, Arrow, K.J. and Pines, D. (eds.), (1988), The Economy as an Evolving Complex System II Addison-Wesley, Reading, MA.

[7] Anderson, S., de Palma, A. and Thisse, J., (1993), Discrete choice theory of product differentiation, MIT Press, Cambridge.

[8] Anufriev, M. (2008), Wealth driven competition in a speculative financial market: examples with maximizing agents, Quantitative Finance, forthcoming.

[9] Anufriev, M. and Bottazzi (2006), Equilibria, stability and asymptotic dominace in a speculative market with heterogeneous agents, Journal of Economic Dynamics and Control 30, 1787-1835.

[10] Anufriev, M. and Hommes, C.H., (2007), Evolution of market heuristics, CeNDEF working paper 07-06, University of Amsterdam.

[11] Arthur, W.B., (1995) Complexity in economic and financial markets, Complexity $1,20-25$.

[12] Arthur, W.B., Durlauf, S.N and Lane, D.A. (eds.), (1997a), The economy as an evolving complex system II Addison-Wesley, Reading, MA.

[13] Arthur, W.B., Holland, J.H., LeBaron, B., Palmer, R. and Tayler, P., (1997b) Asset pricing under endogenous expectations in an artificial stock market, in Arthur, W., Lane, D. and Durlauf, S., (eds.) The economy as an evolving complex system II, Addison-Wesley, pp.15-44.

[14] Baak, S.J. (1999), Tests for bounded rationality with a linear dynamic model 
distorted by heterogeneous expectations, Journal of Economic Dynamics and Control 23, 1517-1543.

[15] Barberis, N. and Thaler, R. (2003), A survey of behavioral finance, Handbook of the Economics of Finance, Edited by G.M. Constantinidis, M. Harris and R. Stulz, Elsevier, pp.1051-1121.

[16] Barberis, N., Shleifer, A. and Vishny, R. (1998), A model of investor sentiment, Journal of Financial Economics 49, 307-343.

[17] Benartzi, S., and Thaler, R.H., Heuristics and Biases in Retirement Savings Behavior, Journal of Economic Perspectives, Forthcoming .

[18] Blume, L. and Easley, D., (1992), Evolution and Market Behavior, Journal of Economic Theory, 58, 9-40.

[19] Blume, L. and Easley, D., (2006), If You're So Smart, Why Aren't You Rich? Belief Selection in Complete and Incomplete Markets, Econometrica 74, 929966.

[20] Böhm, V. and Wenzelburger, J. (2005), On the performance of efficient portfolios, Journal of Economic Dynamics and Control 29, 721-740.

[21] Boswijk, H.P., Hommes, C.H. and Manzan, S. (2007), Behavioral heterogeneity in stock prices, Journal of Economic Dynamics and Control 31, 1938-1970.

[22] Branch, W.A. (2004), The theory of rationally heterogeneous expectations: evidence from survey data on inflation expectations, Economic Journal 114, 592-621.

[23] Branch, W.A. and Evans, G.W., (2006), Intrinsic heterogeneity in expectation formation, Journal of Economic Theory 127, 264-295.

[24] Branch, W.A. and McGough (2008), A new Keynesian model with heterogeneous expectations, working paper University of California, Irvine.

[25] Brock, W.A., (1993) Pathways to randomness in the economy: emergent nonlinearity and chaos in economics and finance, Estudios Económicos 8, $3-55$.

[26] Brock, W.A., (1997), Asset Price Behavior in Complex Environments, in: Arthur, W.B., Durlauf, S.N., and Lane, D.A., eds., The Economy as an Evolving Complex System II, Addison-Wesley, Reading, MA, 385-423.

[27] Brock, W.A., and Hommes, C.H., (1997a) A rational route to randomness, Econometrica 65, 1059-1095.

[28] Brock, W.A., and Hommes, C.H., (1997b) Models of complexity in economics and finance, In: Hey, C. et al. (eds.), System Dynamics in Economic and Financial Models, Chapter 1, Wiley Publ., pp. 3-41.

[29] Brock, W.A., and Hommes, C.H., (1998), Heterogeneous beliefs and routes to chaos in a simple asset pricing model, Journal of Economic Dynamics and Control 22, 1235-1274.

[30] Brock, W.A., and Hommes, C.H., (1999), Rational Animal Spirits, In: Herings, P.J.J., Laan, van der G. and Talman, A.J.J. eds., The Theory of Markets, North-Holland, Amsterdam, 109-137.

[31] Brock, W.A., Hommes, C.H., and Wagener, F.O.O. (2005), Evolutionary 
dynamics in markets with many trader types, Journal of Mathematical Economics, 41, 7-42.

[32] Brock, W.A., Hommes, C.H., and Wagener, F.O.O. (2008), More hedging instruments may destabilize markets, CeNDEF working paper, University of Amsterdam.

[33] Brock, W.A. and LeBaron, (1996), A structural model for stock return volatility and trading volume, Review of Economics and Statistics 78, 94-110.

[34] Brooks, C. and Katsaris, A., (2005), A three-regime model of speculative behaviour: Modelling the evolution of the S\&P500 Composite Index, Economic Journal 115, 767-797.

[35] Campbell, J.Y., Lo, A.W., MacKinlay, A.C., (1997), The econometrics of financial markets, Princeton University Press, Princeton.

[36] Campbell, J.Y. and Shiller, R.J. (2005), Valuation ratios and the long-run stock market outlook: an update, In; Thaler, R.H (Ed.) Advances in Behavioural Finance Vol. 2, Princeton University Press, pp. 173-201.

[37] Chang, S.K, (2007), A simple asset pricing model with social interactions and heterogeneous beliefs, Journal of Economic Dynamics and Control 31, $1300-1325$.

[38] Chavas, J.P., (2000), On information and market dynamics: the case of the U.S. beef market, Journal of Economic Dynamics and Control 24, 833-853.

[39] Chevalier, J. and Ellison, G. (1997), Risk Taking by Mutual Funds as a Response to Incentives, Journal of Political Economy 105, 1167-1200.

[40] Chiarella, C., (2009), survey on heterogeneous agent modeling, this Handbook.

[41] Chiarella, C. and He, X. (2001), Asset price and wealth dynamics under heterogeneous expectations, Quantitative Finance 1, 509-526.

[42] Chiarella, C. and He, X. (2002), Heterogeneous beliefs, risk and learning in a simple asset pricing model, Computational Economics 19, 95-132.

[43] Chiarella, C. and He, X. (2003), Heterogeneous beliefs, risk and learning in a simple asset pricing model with a market maker, Macroeconomic Dynamics $7,503-536$.

[44] Chiarella, C., Dieci, R. and Gardini, L. (2002), Speculative behaviour and complex asset price dynamics: a global analysis, Journal of Economic Behavior \& Organization 49, 173-197.

[45] Chiarella, C., Dieci, R. and Gardini, L. (2006), Asset price and wealth dynamics in a financial market with heterogeneous agents, Journal of Economic Dynamics and Control 30, 1755-1786.

[46] Chiarella, C, Dieci, R. and He, X-Z. (2007), Heterogeneous expectations and speculative behavior in a dynamic multi-asset framework, Journal of Economic Behavior and Organization 62, 408-427.

[47] Cont, R. and Bouchaud, J.-P. (2000), Herd behavior and aggregate fluctuations in financial markets, Macroeconomic Dynamics 4, 170-196.

[48] Cutler, D.M., Poterba, J.M. and Summers, L.H. (1989), What moves stock prices?, Journal of Portfolio Management 15, 4-12. 
[49] DeBondt, W.F.M and Thaler, R.H. (1985), Does the stock market overreact? Journal of Finance 40, 793-805.

[50] DeGrauwe, P., Dewachter, H. and Embrechts, M., (1993) Exchange rate theory. Chaotic models of foreign exchange markets, Blackwell.

[51] DeGrauwe, P. and Grimaldi, M. (2005), Heterogeneity of agents, transaction costs and the exchange rate, Journal of Economic Dynamics and Control 29, 691-719.

[52] DeGrauwe, P. and Grimaldi, M. (2006), Exchange rate puzzles: A tale of switching attractors, European Economic Review 50, 1-33.

[53] Del Guercio, D.D. and Tkac, P.A. (2002), The determinants of the flow of funds of managed portfolios: Mutual funds vs. pension funds Journal of Financial and Quantitative Analysis 37, 523-557.

[54] DeLong, J.B., Shleifer, A., Summers,L.H. and Waldmann, R.J., (1990a) Noise trader risk in financial markets, Journal of Political Economy 98, 703-738.

[55] DeLong, J.B., Shleifer, A., Summers,L.H. and Waldmann, R.J., (1990b) Positive feedback investment strategies and destabilizing rational speculation, Journal of Finance 45, 379-395.

[56] Diks, C.G.H. and Weide, R. van der, (2003), Heterogeneity as a natural source of randomness, CeNDEF Working paper 03-05, University of Amsterdam

[57] Diks, C.G.H. and Weide, R. van der, (2005), Herding, A-synchronous Updating and Heterogeneity in Memory in a CBS, Journal of Economic Dynamics and Control 29, 741-763.

[58] Duffy, J. (2006), Agent-based models and human subject experiments, In: L. Tesfatsion and K.L. Judd (Eds.), Handbook of Computational Economics, Volume 2: Agent-based computational cconomics, Handbooks in Economics Series, Elsevier, Amsterdam, 949-1011.

[59] Duffy, J. (2007), Experimental macroeconomics, In: Blume L, Durlauf S (Eds.), New Palgrave Dictionary of Economics, Macmillan, in press.

[60] Dwyer, G.P., Williams, A.W., Battalio, R.C., Mason, T.I. (1993), Tests of rational expectations in a stark setting, Economic Journal, 103, 586-601.

[61] Evstigneev, I., Hens, T., Reiner Schenk-Hoppé, K., (2002), Market selection of financial trading strategies: global stability, Mathematical Finance 12, 329339.

[62] Evstigneev, I., Hens, T., Reiner Schenk-Hoppé, K., (2008), Globally evolutionary stable portfolio rules, Journal of Economic Theory, forthcoming.

[63] Evstigneev, I., Hens, T., Reiner Schenk-Hoppé, K. (2009), Evolutionary finance, this Handbook.

[64] Fama, E.F., (1970) Efficient capital markets: a review of theory and empirical work, Journal of Finance 25, 383-423.

[65] Fama, E.F. and French, K.R. (2002), The equity premium, Journal of Finance $57,637-659$.

[66] Farmer, J.D., (2002), Market force, ecology, and evolution, Industrial and Corporate Change 11,895-953. 
[67] Farmer, J.D. and Joshi, S., (2002), The price dynamics of common trading strategies, Journal of Economic Behavior \& Organization 49, 149-171.

[68] Fisher, K.L. and Statman, M., (2002), Blowing bubbles, Journal of Psychology and Financial Markets 3, 53-65.

[69] Föllmer, H., Horst, U. and Kirman, A. (2005), Equilibria in financial markets with heterogeneous agents: a probabilistic perspective, Journal of Mathematical Economics 41, 123-155.

[70] de Fontnouvelle, P., (2000), Information dynamics in financial markets, Macroeconomic Dynamics 4, 139-169.

[71] Frankel, J.A. and Froot, K.A., (1987a) Using Survey Data to Test Standard Propositions regarding Exchange Rate Expectations, American Economic Review 77, 133-153.

[72] Frankel, J.A. and Froot, K.A., (1987b) Short-term and Long-term Expectations of the Yen/Dollar Exchange Rate: Evidence from Survey Data, Journal of the Japanese and International Economies 1, 249-274; also published as NBER working paper 2216, April 1987.

[73] Frankel, J.A. and Froot, K.A., (1990a) Chartists, Fundamentalists and the Demand for Dollars, In: Courakis, A.S. and Taylor, M.P. (eds.), Private behaviour and government policy in interdependent economies, New York, Oxford University Press, pp. 73-126. (also published as NBER Working Paper No. r1655, October 1991)

[74] Frankel, J.A. and Froot, K.A., (1990b) The Rationality of the Foreign Exchange Rate. Chartists, Fundamentalists and Trading in the Foreign Exchange Market, American Economic Review 80, no. 2, AEA Papers and Proceedings, 181-185.

[75] Friedman, M., (1953) The case of flexible exchange rates, In: Essays in positive economics, Univ. Chicago Press.

[76] Gaunersdorfer, A., (2000) Endogenous fluctuations in a simple asset pricing model with heterogeneous beliefs, Journal of Economic Dynamics and Control 24, pp.799-831.

[77] Gaunersdorfer, A. and Hommes, C.H., (2007), A nonlinear structural model for volatility clustering, forthcoming in: Kirman, A. and Teyssière, G., (eds.), Microeconomic models for long memory in economics, Springer Verlag, pp. 265-288.

[78] Gaunersdorfer, A., Hommes, C.H., Wagener, F.O.O. (2008), Bifurcation routes to volatility clustering under evolutionary learning, Journal of Economic Behavior and Organization, in press.

[79] Gerber, A., Hens, T., Vogt, B., (2002), Rational investor sentiment, Working Paper 126, Institute for Empirical Research in Economics, University of Zurich.

[80] Gervais, S. and Odean, T. (2001), Learning to be overconfident, Review of Financial Studies 14, 1-27.

[81] Gilli, M. and Winker, P. (2003, A global optimization heuristic for estimating 
agent based models, Computational Statistics \& Data Analysis 42, 299-312.

[82] Goldbaum, D. (2005), Market efficiency and learning in an endogenously unstable environment, Journal of Economic Dynamics and Control 29, 953978.

[83] Gordon, M. (1962), The investment financing and valuation of the corporation, Irwin, Homewood.

[84] Heemeijer, P. (2007), Learning to forecast experiments, Ph-D thesis, Tinbergen Institute Research Series, University of Amsterdam.

[85] Hens, T. and Schenk-Hoppé, K.R. (2005), Evolutionary stability of portfolio rules in incomplete markets, Journal of Mathematical Economics 41, 43-66.

[86] Hey, J.D. (1994), Expectations formation: Rational or adaptive or ... ?, Journal of Economic Behavior \& Organization 25, 329-349.

[87] Hirschleifer, D. (2001), Investor psychology and asset pricing, Journal of Finance 56, 1533-1597.

[88] Hommes, C.H., (2001), Financial markets as nonlinear adaptive evolutionary systems, Quantitative Finance 1, 149-167.

[89] Hommes, C.H., (2002), Modeling the stylized facts in finance through simple nonlinear adaptive systems, Proceedings of the National Academy of Sciences 99, 7221-7228.

[90] Hommes, C.H., 2006. Heterogeneous agent models in economics and finance, In: Tesfatsion, L. and Judd, K.J. (Eds.), Handbook of Computational Economics, Vol. 2: Agent-Based Computational Economics, Elsevier, pp.11091186.

[91] Hommes, C.H., Huang, H. and Wang, D. (2005), A robust rational route to randomness in a simple asset pricing model. Journal of Economic Dynamics and Control 29, 1043-1072.

[92] Hommes, C.H., Sonnemans, J., Tuinstra, J., and van de Velden, H. (2005) Coordination of expectations in asset pricing experiments, Review of Financial Studies 18, 955-980.

[93] Hommes, C.H., Sonnemans, J., Tuinstra, J., and van de Velden, H. (2008) Expectations and bubbles in asset pricing experiments, Journal of Economic Behavior and Organization, in press.

[94] Hong, H. and Stein, J., (1999), A unified theory of underreaction, momentum trading and overreaction in asset markets, Journal of Finance 55, 265-295.

[95] Hong, H. and Stein, J., (2003), Differences of opinion, short-sales constraints, and market crashes, Review of Financial Studies 16, 487-525.

[96] Iori, G. (2002), A microsimulation of traders activity in the stock market: the role of heterogeneity, agents' interactions and trade frictions, Journal of Economic Behavior \& Organization 49, 269-285.

[97] Ippolito, R.A. (1992), Consumer reaction to measures of poor quality: evidence from the mutual fund industry Journal of Law and Economics 35, 45-70.

[98] Ito, K. (1990), Foreign exchange rate expectations, American Economic Re- 
view 80, 434-449.

[99] Kahneman, D. (2003), Maps of bounded rationality: Psychology for behavioral economics, American Economic Review 93, 1449-1475.

[100] Kahneman, D. and Tversky, A. (1973), On the psychology of prediction, Psychological Review 80, 237-251.

[101] Kantz, H. and Schreiber, T. (1997), Nonlinear time series analysis, Cambridge University Press, Cambridge.

[102] Karceski, J. (2002), Returns-chasing behavior, mutual funds and beta's death, Journal of Financial and Quantitative Analysis 37, 559-594.

[103] Kelley, H. and Friedman, D. (2002), Learning to forecast price, Economic Enquiry 40, 556-573.

[104] Kirman, A., (1991) Epidemics of opinion and speculative bubbles in financial markets, In M. Taylor (ed.), Money and financial markets, Macmillan.

[105] Kirman, A., (1993) Ants, rationality and recruitment, Quarterly Journal of Economics 108, 137-156.

[106] Kirman, A. and Teyssière, G., (2002), Microeconomic models for long memory in the volatility of financial time series, Studies in Nonlinear Dynamics \& Econometrics Vol. 5 (4), 281-302.

[107] Kuznetsov, Y. (1995), Elements of applied bifurcation theory, Springer Verlag, New York.

[108] Kyle, A. and Xiong, W. (2001), Contagion as a wealth effect, Journal of Finance 56, 14011440.

[109] LeBaron, B., (2000), Agent based computational finance: suggested readings and early research, Journal of Economic Dynamics and Control 24, 679-702.

[110] LeBaron, B., (2006), Agent-based Computational Finance, In: Tesfatsion, L. and Judd, K.J. (Eds.), Handbook of Computational Economics, Vol. 2: Agent-Based Computational Economics, Elsevier, pp.1187-1232.

[111] LeBaron, B., Arthur, W.B. and Palmer, R. (1999) Time series properties of an artificial stock market, Journal of Economic Dynamics and Control 23, $1487-1516$.

[112] Levy, M., Levy, H. and Solomon, S. (1994), A microscopic model of the stock market, Economics Letters 45, 103-111.

[113] Lucas, R.E., (1971), Econometric testing of the natural rate hypothesis, In: O. Eckstein (ed.) The econometrics of price determination Conference. Board of Governors of the Federal Reserve System and Social Science Research Council.

[114] Lux, T., (2009) Stochastic behavioral asset pricing models and the stylized facts, this Handbook.

[115] Lux, T. and Marchesi, M. (1999) Scaling and criticality in a stochastic multiagent model of a financial market, Nature Vol. 397, February 1999, 498-500.

[116] Lux, T. and Marchesi, M. (2000) Volatility clustering in financial markets: a micro-simulation of interacting agents, International Journal of Theoretical and Applied Finance 3, 675-702.

[117] Manski, C. and McFadden, D., (eds.) (1981), Structural analysis of discrete 
data with econometric applications, MIT Press, Cambridge MA.

[118] Manzan, S. (2003), Essays in nonlinear economic dynamics, Tinbergen Institute Research Series 317, University of Amsterdam.

[119] Marimon, R., S.E. Spear and S. Sunder, (1993), Expectationally driven market volatility: an experimental study, Journal of Economic Theory, 61, 74103.

[120] Marimon, R. and S. Sunder, (1993), Indeterminacy of equilibria in a hyperinflationary world: experimental evidence, Econometrica, 61, 1073-1107.

[121] Marimon, R. and S. Sunder, (1994), Expectations and learning under alternative monetary regimes: an experimental approach, Economic Theory, 4, 131-162.

[122] Marimon, R. and S. Sunder, 1995, Does a constant money growth rule help stabilize inflation? Carnegie-Rochester Conference Series on Public Policy, 43, 111-156.

[123] Muth, J.F., (1961) Rational expectations and the theory of price movements, Econometrica 29, 315-335.

[124] Rockinger (1996), Determinants of capital flows to mutual funds, Working Paper, HEC School of Management.

[125] Scheinkman, J.A. and Xiong, W. (2004), Heterogeneous beliefs, speculation and trading in financial markets, In: Carmona, R.A. et al. (eds.), ParisPrinceton Lectures in Mathematical Finance 2003, Lecture Notes in Mathematics 1847, Springer Verlag, Berlin, pp.217-250.

[126] Schmalensee, R. (1976), An Experimental Study of Expectation Formation, Econometrica 44, 17-41.

[127] Shiller, R.J., (1984) Stock prices and social dynamics, Brookings Papers in Economic Activity 2, 457-510.

[128] Shiller, R.J., (1987) Investor behavior in the October 1987 stock market crash: survey evidence, NBER working paper No. 2446, November 1987, published in: Shiller, R.J., Market Volatility, MIT Press, Cambridge, 1989, chapter 23.

[129] Shiller, R.J., (1989) Market Volatility, MIT Press, Cambridge.

[130] Shiller, R.J., (1990), Speculative prices and popular models, Journal of Economic Perspectives 4, 55-65.

[131] Shiller, R.J., (2000), Measuring bubble expectations and invester confidence, Journal of Psychology and Financial Markets 1, 49-60.

[132] Shleifer, A. (2000), Inefficient markets, Oxford University Press, Oxford.

[133] Shleifer, A. and Vishny, R.W. (1997), The limits of arbitrage, Journal of Finance 52, 35-55.

[134] Sirri, E.R. and Tufano, P. (1998), Costly search and mutual fund flows, Journal of Finance 53, 1589-1622.

[135] Smith, V., Suchanek, G.L. and Williams, A.W., (1988), Bubbles, crashes and endogenous expectations in experimental spot asset markets, Econometrica $56,1119-1151$.

[136] Summers, L.H. (1986), Does the stock market rationally reflect fundamental 
values?, Journal of Finance 41, 591-602.

[137] Sutan, A. and Willinger, M., (2005), Why do we guess better in negative feedback situations? An experiment of beauty contest games with negative feedback and interior equilibria, Working paper University of Montpellier, December 2005.

[138] Taylor, M.P. and Allen, H., (1992), The use of technical analysis in the foreign exchange market, Journal of International Money and Finance 11, 304-314.

[139] Teräsvirta, T. (1994), Specification, estimation, and evaluation of smooth transition autoregressive models, Journal of the American Statistical Association 89, 208-218.

[140] Tesfatsion, L. and K.J. Judd, (2006), Handbook of Computational Economics, Volume 2: Agent-Based Computational Economics, Elsevier, Amsterdam.

[141] Turnovsky, S.J. (1970), Empirical evidence on the formation of price expectations, Journal of the American Statistical Association 65, 1441-59.

[142] Tversky, A. and Kahneman, D. (1974), Judgment under uncertainty: heuristics and biases, Science 185, 1124-1131.

[143] van de Velden, H., (2001), An experimental approach to expectation formation in dynamic economic systems, Ph-D thesis, Tinbergen Institute Research Series 268, University of Amsterdam.

[144] van Norden, S. and Schaller, H. (1999), Speculative behavior, regime switching, and stock market crashes, In: Rothman, P. (Ed.), Nonlinear time series analysis of economic and financial data, Kluwer, Dordrecht, pp.321-356.

[145] Vissing-Jorgensen, A. (2003), Perspective on behavioral finance: does 'irrationality' disappear with wealth? Evidence from expectations and actions, In: Gertler, M., Rogoff, K. (Eds.) NBER Macroeconomics Annual, MIT Press, Cambridge.

[146] Westerhoff, F.H. (2004), Multi-asset market dynamics, Macroeconomic Dynamics 8, 596-616.

[147] Westerhoff, F.H. and Dieci, R. (2006), The effectiveness of Keynes-Tobin transaction taxes when heterogeneous agents can trade in different markets: a behavioral finance approach, Journal of Economic Dynamics and Control 30, 293-322.

[148] Westerhoff, F.H. and Reitz, S. (2003), Nonlinearities and cyclical behavior: the role of chartists and fundamentalists, Studies in Nonlinear Dynamics 86 Econometrics Vol. 7, Issue 4, article 3.

[149] Williams, A.W., (1987), The formation of price forecasts in experimental markets, Journal of Money, Credit and Banking 19, 1-18.

[150] Winker, P. and Gilli, M. (2001), Indirect estimation of the parameters of agent based models of financial markets, FAME Research Paper No. 38, University of Geneva, November 2001.

[151] Youssefmir, M. and Huberman, B.A., (1997), Clustered volatility in multi agent dynamics, Journal of Economic Behavior $\& 6$ Organization 32, 101-118.

[152] Zwart, G.J., Markwat, T., Swinkels, L.A.P. and van Dijk, D. (2007), Chartists 
and fundamentalists in emerging currency markets, Working Paper, Erasmus School of Economics. 